\title{
Effect of genetic background on the evolution of Vancomycin- Intermediate Staphylococcus aureus (VISA)
}

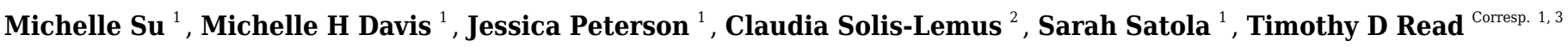 \\ ${ }^{1}$ Division of Infectious Diseases, Department of Medicine, School of Medicine, Emory University, Atlanta, Georgia, USA \\ 2 Wisconsin Institute for Discovery and Department of Plant Pathology, University of Wisconsin-Madison, Madison, Wisconsin, USA \\ 3 Department of Dermatology, School of Medicine, Emory University, Atlanta, Georgia, USA \\ Corresponding Author: Timothy D Read \\ Email address: tread@emory.edu
}

Vancomycin-intermediate Staphylococcus aureus (VISA) typically arises through accumulation of chromosomal mutations that alter cell-wall thickness and global regulatory pathways. Genome-based prediction of VISA requires understanding whether strain background influences patterns of mutation that lead to resistance. We used an iterative method to experimentally evolve three important methicillin-resistant $S$. aureus (MRSA) strain backgrounds - (CC1, CC5 and CC8 (USA300)) to generate a library of 120 laboratory selected VISA isolates. At the endpoint, isolates had vancomycin MICs ranging from 4 to $10 \mu \mathrm{g} / \mathrm{mL}$. We detected mutations in more than 150 genes, but only six genes (already known to be associated with VISA from prior studies) were mutated in all three background strains (walK, prs, rpoB, rpoC, vraS, yvqF). We found evidence of interactions between loci (e.g vraS and $y v q F$ mutants were significantly negatively correlated) and $r p o B, r p o C$, vraS and $y v q F$ were more frequently mutated in one of the backgrounds. Increasing vancomycin resistance was correlated with lower maximal growth rates (a proxy for fitness) regardless of background. However, CC5 VISA isolates had higher MICS with fewer rounds of selection and had lower fitness costs than the CC8 VISA isolates. Using multivariable regression, we found that genes differed in their contribution to overall MIC depending on the background. Overall, these results demonstrated that VISA evolved through mutations in a similar set of loci in all backgrounds, but the effect of mutation in common genes differed with regard to fitness and contribution to resistance in different strains. 
2 Effect of genetic background on the evolution of

3 Vancomycin-Intermediate Staphylococcus aureus 4 (VISA)

Michelle Su${ }^{1}$, Michelle H. Davis ${ }^{1}$, Jessica Peterson ${ }^{1}$, Claudia Solís-Lemus ${ }^{2}$, Sarah W. Satola ${ }^{1}$, Timothy D. Read ${ }^{1,3}$

${ }^{1}$ Division of Infectious Diseases, Department of Medicine, School of Medicine, Emory University, Atlanta, GA, USA

${ }^{2}$ Wisconsin Institute for Discovery and Department of Plant Pathology, University of

Wisconsin-Madison, Madison, WI, USA

${ }^{3}$ Department of Human Genetics, School of Medicine, Emory University, Atlanta, GA, USA

15

Corresponding Author:

Timothy D. Read ${ }^{1,3}$

1760 Haygood Dr E130, Atlanta, Georgia, 30322, USA

Email address: tread@emory.edu

\section{Abstract}

Vancomycin-intermediate Staphylococcus aureus (VISA) typically arises through accumulation of chromosomal mutations that alter cell-wall thickness and global regulatory pathways. patterns of mutation that lead to resistance. We used an iterative method to experimentally evolve three important methicillin-resistant $S$. aureus (MRSA) strain backgrounds - (CC1, CC5 and CC8 (USA300)) to generate a library of 120 laboratory selected VISA isolates. At the endpoint, isolates had vancomycin MICs ranging from 4 to $10 \mu \mathrm{g} / \mathrm{mL}$. We detected mutations in more than 150 genes, but only six genes (already known to be associated with VISA from prior studies) were mutated in all three background strains (walK, prs, rpoB, rpoC, vraS, yvqF). We found evidence of interactions between loci (e.g., $v r a S$ and $y v q F$ mutants were significantly negatively correlated), and $r p o B, r p o C, v r a S$, and $y v q F$ were more frequently mutated in one of the backgrounds. Increasing vancomycin resistance was correlated with lower maximal growth rates (a proxy for fitness) regardless of background. However, CC5 VISA isolates had higher MICs with fewer rounds of selection and had lower fitness costs than the CC8 VISA isolates. Using multivariable regression, we found that genes differed in their contribution to overall MIC depending on the background. Overall, these results demonstrated that VISA evolved through 
38

39

40

41

42

43

44

45

46

47

48

49

50

51

52

53

54

55

56

57

58

59

60

61

62

63

64

65

66

67

68

69

70

71

72

73

74

75

76

77

mutations in a similar set of loci in all backgrounds, but the effect of mutation in common genes differed with regard to fitness and contribution to resistance in different strains.

\section{Introduction}

Vancomycin has been a relatively safe and economical drug against MRSA (methicillin-resistant Staphylococcus aureus) for the past three decades but there have been numerous reports of strains with reduced susceptibility (which we will call "vancomycin-resistant", using the common terminology) (Gardete \& Tomasz, 2014; Zhang et al., 2015; McGuinness, Malachowa \& DeLeo, 2017). High-level vancomycin-resistant S. aureus (VRSA; minimum inhibitory concentrations (MIC) $\geq 16 \mu \mathrm{g} / \mathrm{mL}$ ) strains have acquired the vanA gene from horizontal transfer from Enterococcus spp (Kobayashi, Musser \& DeLeo, 2012). The incidence of VRSA has remained rare, likely due to poor regulation of expression of the vanA gene in $S$. aureus imposing a fitness burden on the bacterium (Foucault, Courvalin \& Grillot-Courvalin, 2009). Vancomycinintermediate (VISA; MIC 4-8 $\mu \mathrm{g} / \mathrm{mL}$ ) strains, first reported in 1997 (Hiramatsu et al., 1997), are more commonly encountered in the clinic than VRSA. VISA evolves from vancomycinsusceptible $S$. aureus (VSSA; MIC $\leq 2 \mu \mathrm{g} / \mathrm{mL}$ ) through intrinsic mutagenesis. VISA strains susceptible in vitro to vancomycin (MIC $\leq 2 \mu \mathrm{g} / \mathrm{mL}$ ) but containing subpopulations that can grow in the presence of $\geq 4 \mu \mathrm{g} / \mathrm{mL}$ of vancomycin and thus are capable of spontaneous transitions to VISA are termed hVISA (for heterogeneous VISA) (Liu \& Chambers, 2003; Sakoulas \& Moellering, 2008; Deresinski, 2009; El-Halfawy \& Valvano, 2015).

Most intermediate resistance is acquired by within-patient evolution (Klevens et al., 2007; Gardete \& Tomasz, 2014). The classic genomics study by Mwangi et al (Mwangi et al., 2007) showed VISA evolving within a patient on long-term vancomycin therapy through a series of adaptive mutations. VISA has emerged independently in strains from each of the major MRSA lineages (Hiramatsu et al., 1997; Howden et al., 2006, 2008; Klevens et al., 2007; Mwangi et al., 2007; Alam et al., 2014; Gardete \& Tomasz, 2014). Some patients with MRSA infections failing therapy showed enhanced vancomycin MIC even when the drug was not used (Horne et al., 2009; Lalueza et al., 2010; Holland \& Fowler, 2011), suggesting that VISA may overlap with 'persister' phenotypes (Johnson \& Levin, 2013) associated with long-term invasive infection.

Standard microbiology laboratory-based phenotypic tests for VISA/hVISA are labor intensive, and the incidence may be higher than currently reported (Marlowe et al., 2001; Charles et al., 2004; Prakash, V, Lewis \& Jorgensen, 2008; Satola et al., 2009; Swenson et al., 2009; Vaudaux et al., 2010). hVISA strains are particularly challenging to detect using current standard clinical microbiology methods (automated broth microdilution assays) as MICs can overlap with VSSA (Swenson et al., 2009). Development of a nucleic acid test using standard PCR based approaches has been confounded to date by the complexity of the genetics of VISA. The genetic basis of VISA has been investigated for several years, primarily through identification of mutations by comparative sequencing of small numbers of isogenic clinical samples (Ohta et al., 2004; 
79

80

81

82

83

84

85

86

87

88

89

90

91

92

93

94

95

96

97

98

99

100

101

102

103

104

105

106

107

108

109

110

111

112

113

114

115

116

117

Mwangi et al., 2007; Howden et al., 2008) and molecular genetic characterization. VISA cells typically show cell-wall thickening and a reduction of fitness in growth compared to isogenic parents. Mutations in a variety of conserved core $S$. aureus genes have been reported to be associated with VISA (Howden et al., 2010; Gardete \& Tomasz, 2014; Wang et al., 2016), most commonly those involved in regulation of cell wall architecture (e.g., graRS (Neoh et al., 2008; Howden et al., 2008), vraRS (Kato et al., 2010; Baek et al., 2017; Asadpour \& Ghazanfari, 2019), yvqF (Kato et al., 2010; Yoo et al., 2013), walK (Shoji et al., 2011; Hafer et al., 2012; McEvoy et al., 2013; Hu et al., 2015), walR (Howden et al., 2011)) or certain global transcriptional regulators (e.g., agr (Sakoulas et al., 2003), stp1 (Cameron et al., 2012; Passalacqua et al., 2012), rpoB (Cui et al., 2010; Watanabe et al., 2011; Hafer et al., 2012; Katayama et al., 2017) and rpoC (Matsuo et al., 2013)). walK controls cell wall autolysis while $v r a S$ and $y v q F$ (also known as $v r a T$ ) regulate cell wall synthesis, thus mutations in these genes contribute to the characteristic thick cell wall of VISA strains.

It is vital for any future gene-based test for VISA to know if there are epistatic interactions between $S$. aureus strain background and drug resistance mutations. Recent publications have reported important epistatic interactions that drive patterns of antibiotic-resistance (Schubert et al., 2018; Ma et al., 2020). There are also examples of strain-specific effects in S. aureus: clinical CC30 S. aureus strains were found to have elevated (average 100 fold higher) persister formation compared to CC5, CC8, CC30, and CC45 strains (Liu et al., 2020), and under experimental evolution, CC398 strains were found to frequently evolve ciprofloxacin resistance due to a lineage-specific IS element that allowed amplification of the nor $A$ efflux pump (Papkou et al., 2020). In this study, we aimed to evaluate the relationship between genetic determinants of intermediate vancomycin resistance and strain background to determine if epistasis can direct the evolutionary trajectory of this phenotype. We evolved VISA strains from three common genetic backgrounds encountered clinically and analyzed the differences in mutation, fitness, and resistance levels.

\section{Materials \& Methods}

\section{Strains}

Strains used for the evolution experiments: NRS70 (N315), NRS123 (MW2), and NRS384 (USA300-0114), were obtained from BEI resources (https://www.beiresources.org/).

\section{Experimental Evolution of vancomycin resistance}

The strategy for the evolution experiment is outlined in Figure 1. Parent strains were streaked on Brain Heart Infusion (BHI) plates from frozen stocks, and single colonies used to establish shaking overnight cultures of BHI broth. We then propagated independent lines in BHI broth shaking at $250 \mathrm{rpm}$ at $37^{\circ} \mathrm{C}$ until cultures were turbid (OD600 >1.5) before diluting cultures at a 1:500 dilution into fresh media. Strains were grown initially on BHI broth containing $1 \mu \mathrm{g} / \mathrm{mL}$ vancomycin then transferred to BHI broth with $2 \mu \mathrm{g} / \mathrm{mL}$ antibiotic. Once turbid, the cultures 
118 were similarly diluted to $4 \mu \mathrm{g} / \mathrm{mL}$, then grown for 3 days before transfer to $8 \mu \mathrm{g} / \mathrm{mL}$, and grown 119 for a final 3 days. After growth in broth at the final antibiotic concentration, cultures were plated 120 on BHI plates containing 2,4 , and $8 \mu \mathrm{g} / \mathrm{mL}$ of vancomycin. A single colony was picked from the 121 highest concentration at 48 hours growth. The colony was passaged twice on selective media and 122 then examined by Gram stain to confirm species.

123

124

125

126

127

128

129

130

131

132

133

134

135

136

137

138

139

140

141

142

143

144

145

146

147

148

149

150

151

152

153

154

155

156

\section{Genomic Analysis}

DNA extraction and library prep were performed as manufacturer's instructions (Wizard Genomic DNA Purification Kit, Promega; Nextera XT DNA Library Prep Kit, Illumina). Genome sequencing was performed on Illumina HiSeq and MiSeq with paired-end reads. Raw read data were deposited in the NCBI Short Read Archive under project accession number PRJNA525705.

Raw read data was processed by BBduk (v37.66) to filter out adapters associated with Illumina sequencing and trim reads based on quality. Mutations were called based on comparison to reference sequences using breseq (Deatherage \& Barrick, 2014) CONSENSUS mode. Reference genomes used for NRS70, NRS123, and NRS384 VISA strains were NC_002745.2 (BioProject: PRJNA224116, BioSample: SAMD00061099, Assembly: GCF_000009645.1), NC_003923.1

(BioProject: PRJNA224116, BioSample: SAMD00061104, Assembly: GCF_000011265.1), and NZ_CP027476.1 (BioProject: PRJNA224116, BioSample: SAMN07411405, Assembly: GCF_002993865.1) respectively. To compare mutations across the three strains, the protein sequences of NRS123 and NRS384 were aligned using BLAST against that of NRS70 (Camacho et al., 2009). Genes were considered orthologous to NRS70 if the amino acid sequences had at least $95 \%$ identity, $95 \%$ coverage, or an evalue $=0$. To determine if large scale deletions were due to phage loss, PhageWeb was used to identify prophages within the genomes (Sousa et al., 2018).

\section{Measuring fitness based on growth}

Strains were grown in duplicate in a 96-well plate beginning at an OD $<.1$ and grown for 24 hours at $37^{\circ} \mathrm{C}$ with constant shaking in a Biotek Eon Microplate Spectrophotometer, with OD measurements every 10 minutes. To assess growth curves, OD readings were imported into $\mathrm{R} \underline{\mathrm{R}}$ Core Team, R Foundation for Statistical Computing, Vienna, Austria., 2016) and maximal growth rate $(r)$ calculated using the growthcurver package (Sprouffske \& Wagner, 2016) using the average of two biological replicates. Fitness was calculated as the ratio between the average $r$ of each evolved strain to the average $r$ of the parent strain. Fitness distributions between NRS70 evolved VISA strains and NRS384 evolved VISA strains were compared using a two-sided twosample Kolmogorov-Smirnov test.

\section{Statistical analysis}


157 To determine if the difference in the prevalence of mutations across different VISA genes (walK,

158

159

160

161

162

163

164

165

166

167

168

169

170

171

172

173

174

175

176

177

178

179

180

181

182

183

184

185

186

187

188

189

190

191

192

193

194

195

196

prs, rpoB, rpoC, vraS, $y v q F$ ) was significant, a Pearson's Chi-squared test was performed, with subsequent post hoc pairwise comparisons using Fisher's exact test and a Bonferroni multiple test correction. To analyze if the prevalence of mutations was also affected by background, binomial generalized linear models (GLMs) were fitted for each gene, and pairwise Wald tests were performed to test whether the difference in the coefficients for each background was nonzero. The statistical significance of gene correlation was assessed using a Spearman correlation with a Holm multiple testing correction. Total SNP distributions were compared using a KruskalWallis test followed by pairwise Mann-Whitney U tests. Synonymous and nonsynonymous SNP distributions were compared with a Pearson's Chi-squared test followed by Bonferroni corrected Fisher's exact tests. The vancomycin and daptomycin MIC distributions between NRS70 VISA strains and NRS384 VISA strains were compared using two-sided two-sample KolmogorovSmirnov tests. To test if the linear relationship between vancomycin MIC and daptomycin MIC was dependent on background (NRS70 and NRS384), an Analysis of Covariance (ANCOVA) was performed.

To assess the effect of mutations in individual genes on vancomycin MIC, linear regression models were fitted using the $l m$ function in R. walK, prs, rpoB, rpoC, vraS, and $y v q F$ as binary variables (mutated or not) were used as predictors for log transformed vancomycin MICs. All mutations found in these genes were classified as nonsynonymous SNPs. The final model was chosen by backwards selection with the goal of minimizing Akaike information criterion (AIC) as in Eyre et al (Eyre et al., 2017).

To investigate differences in the effect sizes of SNPs on vancomycin MIC from background, standard linear models were fitted with interaction terms for SNP presence and background. In these models, strain background was included as a fixed-effects predictor because the mixedeffects version (with background as a vector random effect affecting the main and interaction effects of the SNPs) was not computationally stable. To study potential pairwise interactions between SNPs affecting the MIC of vancomycin, linear mixed-effects models were fit with presence or absence of SNPs as fixed-effects predictors (main and interaction effects) and with background (NRS70, NRS384) as a scalar random effect. Mixed-effects models with vector random effects (affecting intercept and slope of certain interactions) were also tested, but these models were not stable enough for convergence. Given the sparsity of the data matrix, the predictors were restricted to SNPs present in at least $10 \%$ of the strains. Predictors included were the six universally mutated genes (walK, prs, rpoB, rpoC, vraS, $y v q F$ ) and $s d r C$. All SNPs with the exception of SNPs in $s d r C$, which were all synonymous, were nonsynonymous. The response variable (vancomycin MIC) was log transformed. These models were also fitted for another response (log transformed growth rate as a proxy of fitness). Similar to the vancomycin models, pairwise SNP interactions were assessed with mixed-effects models and SNP-background interactions with standard linear models. A Bonferroni corrected $\mathrm{p}$-value $(\mathrm{a}=0.00056)$ was used

PeerJ reviewing PDF | (2020:11:55692:2:1:NEW 17 Jun 2021) 
197 to assess test significance. R scripts used for these analyses are available at

198 https://github.com/crs14/staph-visa

199

200

Antimicrobial susceptibility testing

201

Antimicrobial susceptibility testing for daptomycin, vancomycin, and methicillin were

202

performed according to Clinical Laboratory Standards Institute (CLSI) standards (CLSI, 2013).

203 In brief, cultures were streaked from a frozen stock onto BHI agar and restreaked the next day.

204 From the second day culture, colonies were resuspended in normal saline to a $0.5 \mathrm{McFarland}$

205 standard. For plate-based testing, e-test for daptomycin and disc diffusion with cefoxitin, cultures

206 were struck to create a lawn on cation-adjusted Mueller-Hinton broth (CAMHB) agar plates

207 before application. Plates were incubated at $35^{\circ} \mathrm{C}$ and read at 18 hours. For broth microdilution

208 (BMD) to determine strain vancomycin minimum inhibitory concentration (MIC), 0.5

209 McFarland standards were diluted 1:20 in normal saline, and 10 $\mu 1$ was added to $90 \mu 1$ of

210 $\mathrm{CAMHB}$ with the appropriate concentration of vancomycin. Plates were incubated at $35^{\circ} \mathrm{C}$

211 without shaking and read at 24 hours.

212

213

\section{Results}

215

Patterns of acquisition of SNPs at VISA endpoints is influenced by genetic background

216

Three strains, representing major clinical lineages in the US: N315/NRS70 (Kuroda et al., 2001)

217 (ST5/CC5), USA300-0114/NRS384 (Diep et al., 2006) (ST8/CC8) and MW2/NRS123 (Baba et

218 al., 2002) (ST1/CC1) were experimentally evolved under selection pressure to achieve a

219 vancomycin MIC of $4-10 \mu \mathrm{g} / \mathrm{mL}$ from an initial MIC of $1 \mu \mathrm{g} / \mathrm{mL}$ (Figure 1). Selection of 120 of

220 these mutants, 40 independent lines per genetic background, was achieved by sequentially

221 raising the vancomycin concentration through serial passages of strains in BHI broth. Mutants

222 were sequenced and compared to their isogenic parent using breseq (Deatherage \& Barrick, 2014) to ascertain mutations acquired during vancomycin selection. Thirteen evolved strains are

223 excluded from further analyses due to either low sequence quality or culture contamination. The

224

225 final number of evolved VISA strains per background with high quality DNA sequences was 35 NRS70 strains, 34 NRS123 strains, and 38 NRS384 strains.

226

227

228

The majority of the genetic changes that occurred during the selection for vancomycin-

229 intermediate resistance were single nucleotide polymorphisms (SNPs) and will be the focus of

230

231 the rest of the analyses. Most deletions, insertions, and substitutions only occurred once.

232 (Supplemental Tables 1-6). Of note, prophage loss (StauST398-4 in NRS70, Pvl108 in NRS123, 23MRA in NRS384) was observed in four strains from each of the genetic

233 backgrounds. Phage loss has been previously observed in vancomycin and daptomycin resistant isolates and has been associated with the induction of the SOS response by antibiotics (Maiques

234 et al., 2006; Boyle-Vavra et al., 2011; Machado et al., 2020).

235 
236 We found between 5 and 25 SNPs in evolved VISA strains compared to their respective parent

237

238

239

240

241

242

243

244

245

246

247

248

249

250

251

252

253

254

255

256

257

258

259

260

261

262

263

264

265

266

267

268

269

270

271

272

273

274

275 strains, including non-synonymous mutations in several genes previously reported to be associated with VISA genes. As the genome sizes for NRS70, NRS123 and NRS384 are 2.81 $\mathrm{Mb}, 2.82 \mathrm{Mb}$, and $2.87 \mathrm{Mb}$, respectively, we did not expect differences in genome size to significantly influence the number of mutations observed during the course of selection. SNPs fell within 151 coding genes, and no gene was universally mutated in all strains, highlighting the existence of multiple pathways to intermediate vancomycin resistance in $S$. aureus. However, of the 151 genes, 121 genes had mutations in only one of the three backgrounds; 24 genes were mutated in two backgrounds; and only six genes (walK, prs, rpoB, $r p o C$, vraS, $y v q F$ ) had non-synonymous mutations in all three backgrounds. The six "universally mutated" genes have been previously implicated in VISA from sequencing of clinical isolates, but interestingly, some genes that had been previously reported did not acquire mutations frequently or in all three backgrounds. Notably, we did not find any agrR mutants, and graRS mutants were found in only NRS384 derived strains and walR (Howden, Peleg \& Stinear, 2013) only in NRS70 and NRS123 derived strains.

The six universally mutated genes varied in their frequency across strains. On opposite sides of the spectrum, mutations in prs occurred in fewer than $10 \%$ of the evolved strains while mutations in walK occurred in 80-95\% of the evolved VISA strains (Table 1). A Pearson's chi-squared test $\left(\mathrm{p}<2.2 \times 10^{-16}\right)$ and post hoc Fisher's exact tests $\left(\right.$ all $\left.\mathrm{p}<5.91 \times 10^{-03}\right)$ determined that the proportion of strains differed significantly between each of these six genes. There was also evidence that some gene mutations occurred more frequently in some backgrounds than others. By fitting binomial generalized linear models (GLMs) to the individual gene mutation distributions with the genetic background as a predictor, proportions of strains with rpoB (NRS70 vs. NRS123, $\mathrm{p}=.03$; NRS70 vs. NRS384, $\mathrm{p}=.015), r p o C(\mathrm{NRS70}$ vs. NRS384, $\mathrm{p}=$ $.019)$, vraS (NRS70 vs. NRS384, $\mathrm{p}=.04$ ), and $y v q F$ (NRS70 vs. NRS123, $\mathrm{p}=.035$; NRS70 vs. $\mathrm{NRS384}, \mathrm{p}=.028$ ) were found to be significantly influenced by genetic background. Between NRS70 and NRS123, five other genes were mutated in both backgrounds. Similarly, between NRS123 and NRS384, six other genes were mutated. Finally, between NRS70 and NRS384, 12 other genes were mutated (Table 2). The higher proportion of other shared genes between NRS70 and NRS384 may indicate that these two strains share more similar evolutionary paths toward vancomycin resistance, but mutations in these genes were rare, and differences were not statistically significant.

We examined the co-occurrence of mutations in the six universally mutated genes (Figure 2a-d). Mutations in $v r a S$ and $y v q F$ were almost mutually exclusive (coefficient $-.8, \mathrm{p}<.0001$ ), with minor differences between genetic backgrounds (Figure 2b-d, NRS70 and NRS384: -.9; NRS123: -.6). NRS70 was the only background where we detected significant associations between other genes (Figure 2b): prs/vraS (coefficient .5, $\mathrm{p}=.011$ ) and prs $/ y v q F$ (coefficient $.5, \mathrm{p}=.035)$.

Peer] reviewing PDF | (2020:11:55692:2:1:NEW 17 Jun 2021) 
276

277

278

279

280

281

282

283

284

285

286

287

288

289

290

291

292

293

294

295

296

297

298

299

300

301

302

303

304

305

306

307

308

309

310

311

312

313

314

315

To examine the distribution of amino acid changes caused by SNPs, we focused on $y v q F$ and walK due to the high frequency of mutation in our VISA strains (Table 1). For $y v q F$, there were 32 SNPs in 68 strains: 3 SNPs are found in all three backgrounds, 10 SNPs are found in two, and 19 SNPs in only one. Despite walK mutations (41 SNPs across 93 strains) being more common, there are no SNPs in common between the three backgrounds, and 31 SNPs are found in only one. Furthermore, the most common SNPs within each background for these two genes were found in different amino acid residues ( $y v q F$ : NRS70/A152V, NRS123/P126S, NRS384/P174A; walK: NRS70/E236D, NRS123/I29M, NRS384/Q216E).

In population genetics, there is a common a priori assumption that synonymous SNPs are regarded as having little or no functional impact compared to non-synonymous. However, we noted some genes with high rates of synonymous mutations. Strikingly, $s d r C$ was mutated in 7 out of 38 NRS384 VISA strains. The $s d r$ locus encodes $s d r C, s d r D$, $s d r E$ which are members of the repeat-rich microbial surface components recognizing adhesive matrix molecules (MSCRAMM) family (Josefsson et al., 1998). These genes are not always conserved together (Liu et al., 2015), and their presence has been associated with bone infection (Sabat et al., 2006), resistance to host immunity (Sitkiewicz, Babiak \& Hryniewicz, 2011; Askarian et al., 2017), biofilm formation (Barbu et al., 2014), and host-cell adhesion (Corrigan, Miajlovic \& Foster, 2009; Cheng et al., 2009; Barbu et al., 2010; Askarian et al., 2016). Belikova et al (Belikova et al., 2020) showed that in USA300 $s d r$ genes undergo frequent within-genome recombination during growth in lab conditions and during infection. The high number of synonymous mutations may be the result of repeats expanding or collapsing in evolved strains, causing base-calling artefacts when mapped onto the reference genomes. Ultimately, long-read sequencing approaches are needed to deconvolute these complex structures, but there is a strong possibility that base-calling artefacts inflated synonymous mutation numbers in some strains. We also noted that there were significant differences between backgrounds in the total number of mutations that accrued during the evolution experiments $\left(\mathrm{p}=1.1 \times 10^{-5}\right)$. NRS123 VISA (median 3 mutations) strains had fewer mutations than NRS70 (median 4, $\mathrm{p}=.004$ ) and NRS384 (median 5, $\mathrm{p}=2.8 \mathrm{x}$ $10^{-5}$ ) VISA strains (Figure 3). Furthermore, the number of synonymous SNPs relative to nonsynonymous SNPs was not the same (Table 3, Pearson chi square $\mathrm{p}=1.8 \times 10^{-5}$, NRS70 vs. NRS123 $\mathrm{p}=.046$, NRS70 vs. NRS384 $\mathrm{p}=.038$, NRS123 vs. NRS384 $\mathrm{p}=1.0 \times 10^{-7}$ ). However, when excluding $s d r$ mutations, NRS70 and NRS123 are no longer statistically significantly different, but NRS384 remained statistically different from NRS70 and NRS123 (Table 3, Pearson chi square $\mathrm{p}=.00021$, NRS70 vs. NRS384 $\mathrm{p}=.014$, NRS123 vs. NRS384 $\mathrm{p}=.0011$ ). Furthermore, the number of synonymous SNPs relative to nonsynonymous SNPs was not the same (Table 3, Pearson chi square $\mathrm{p}=1.8 \times 10^{-5}$, NRS70 vs. NRS123 $\mathrm{p}=.046$, NRS70 vs. NRS384 $\mathrm{p}=.038, \mathrm{NRS} 123$ vs. NRS384 $\left.\mathrm{p}=1.0 \times 10^{-7}\right)$.

\section{Resistance phenotypes of evolved VISA strains}

Peer] reviewing PDF | (2020:11:55692:2:1:NEW 17 Jun 2021) 
316 All evolved VISA strains at the endpoint of the iterative selection were able to grow in $8 \mu \mathrm{g} / \mathrm{mL}$ 317 vancomycin BHI broth. However, to accurately measure the level of resistance, we performed 318 BMD on NRS70 and NRS384 strains according to CLSI standards (unfortunately, all NRS123 319 frozen cultures were accidentally discarded while in $-80^{\circ} \mathrm{C}$ storage before we could test them). 320 All endpoint NRS70 and NRS384 strains had MICs within the range to be considered VISA

321 (Figure 4a), but some (20 NRS70 and 28 NRS384 strains) were found to be below $8 \mu \mathrm{g} / \mathrm{mL}$. The 322 NRS70 VISA strain MICs ranged from 4 to $10 \mu \mathrm{g} / \mathrm{mL}$, with a median and mode of $6 \mu \mathrm{g} / \mathrm{mL}$, 323 while the NRS384 VISA strain MICs ranged from 4 to $8 \mu \mathrm{g} / \mathrm{mL}$, with a median and mode of 6

324

325

326

327

328

329

330

331

332

333

334

335

336

337

338

339

340

341

342

343

344

345

346

347

348

349

350

351

352

353

354

355 $\mu \mathrm{g} / \mathrm{mL}$. The difference in the MIC distributions was not statistically significant between NRS70 and NRS384 strains $(\mathrm{p}=.323)$. Mutated genes and/or mutated gene patterns associated with isolates with the highest level of vancomycin resistance were not appreciably different than those found in isolates with a lower resistance, likely indicating that individual mutations played a large role in the resistance achieved versus genes overall.

The cross-resistance of vancomycin and daptomycin in VISA strains has been well-studied (Cui et al., 2006; Nam et al., 2018). NRS70 and NRS384 endpoint strains were thus tested for development of daptomycin resistance by E-test. Parental strains had daptomycin MICs of 0.38 $\mu \mathrm{g} / \mathrm{mL}$. Thirty of the $35 \mathrm{NRS} 70$ strains became resistant, and 33 of the 38 NRS384 strains became resistant (Figure 4b). As observed with the vancomycin resistance genes, there was no clear mutational pattern associated with higher daptomycin resistance. The daptomycin distributions between NRS70 and NRS384 were not statistically different $(p=1)$, however, only NRS384 strains had a daptomycin MIC of $6 \mu \mathrm{g} / \mathrm{mL}$ versus $4 \mu \mathrm{g} / \mathrm{mL}$ for NRS70 strains. Greater levels of vancomycin resistance correlated to greater levels of daptomycin resistance (Figure 5, $\mathrm{p}=.006)$. However, the regressions for each background were not significantly different $(\mathrm{p}=$ $.822)$.

Collateral sensitivity with beta-lactams is a less frequently reported phenomenon in VISA strains (Sieradzki \& Tomasz, 1999; Mwangi et al., 2007; Barber et al., 2014). Within our evolved NRS70 and NRS384 strains, only two NRS70 strains became methicillin sensitive. Known mutations for beta-lactam sensitivity in a MRSA background such as loss of SCCmec or inactivation of $m e c A$ were not found, and there the mechanism for collateral sensitivity in these strains was unknown.

\section{Fitness decreases during evolution of higher resistance but is modulated by genetic background}

We examined the fitness of intermediate and endpoint strains of each NRS70 and NRS384 experimentally evolved lineage using maximum growth rate in batch culture $\log (\mathrm{r})$ as an indicator. Isolates from later stages of selection, able to tolerate greater concentration of vancomycin, generally equated to lower fitness (Figures 6a-b). Looking at the fitness distributions at each selection stage $(4 \mu \mathrm{g} / \mathrm{mL}, 6 \mu \mathrm{g} / \mathrm{mL}, 8 \mu \mathrm{g} / \mathrm{mL})$ between NRS70 and NRS384, 
356

357

358

359

360

361

362

363

364

365

366

367

368

369

370

371

372

373

374

375

376

377

378

379

380

381

382

383

384

385

386

387

388

389

390

391

392

393

394

395

there was a striking difference at $4 \mu \mathrm{g} / \mathrm{mL}\left(\mathrm{p}=7.08 \times 10^{-6}\right)$ and $6 \mu \mathrm{g} / \mathrm{mL}\left(\mathrm{p}=1.86 \times 10^{-6}\right)$, which disappeared at $8 \mu \mathrm{g} / \mathrm{mL}(\mathrm{p}=.273)$ (Figures 7a-c). Thus, NRS70 strains appeared to be able to tolerate lower levels of vancomycin resistance with lower fitness cost compared to NRS384 strains. Linear mixed models with SNP presence/absence as fixed effects and background as a scalar random effect were used to determine interaction effects between individual SNPs and $\log (\mathrm{r})$. However, no results were statistically significant after Bonferroni correction. The strongest interaction was between $s d r C$ and $r p o C$ whereby mutations in $s d r C$ had a negative effect on growth rate in the presence of $r p o C$ mutation from a previous positive effect (uncorrected $\mathrm{p}=.023$, Figure S1), but as noted above, the caveat is that $s d r C$ mutations may be surrogates for more complex recombination events.

\section{Genetic predictors of vancomycin MIC}

To investigate the interactions of mutation and $S$. aureus strain background to vancomycin resistance, we used two modelling approaches. In the first, we used multiple linear regression models to determine which mutated genes were most predictive of vancomycin resistance. To reduce the number of parameters and for ease of comparison, only the six universally mutated genes were used in the initial models (Table 4). To make the models more meaningful, parameters were evaluated for inclusion in the final models using AIC (Eyre et al., 2017). In the final model for NRS70, walK (coefficient estimate .298), rpoC (.228), vraS (.989), and yvqF (1.058) were statistically significantly associated with vancomycin resistance, defined as $\log (\mathrm{MIC})\left(\mathrm{R}^{2}=.583, \mathrm{p}=2.15 \times 10^{-6}\right)$. In comparison, only walK (coefficient estimate .633) by itself was statistically significant in the final model of NRS384 $\left(\mathrm{R}^{2}=.313, \mathrm{p}=9.95 \times 10^{-4}\right)$, although $v r a S(.340)$ and $y v q F(.378)$ were included for improved model fit.

In the second approach, we fit linear regression models to determine if the effect sizes of SNPs on $\log (\mathrm{MIC})$ differed by background. No results were statistically significant after Bonferroni correction, but there was a suggestive result that $r p o C$ may contribute positively to vancomycin MIC in NRS70 strains but not NRS384 strains (uncorrected $p=0.028$, Figure S2), which is consistent with our previous linear regression models. Linear mixed models were used to determine interaction effects between SNPs. No results were statistically significant after Bonferroni correction, but there was a suggestive result that the effect of a $s d r C$ mutation (either increasing or decreasing vancomycin MIC) depended on walK (uncorrected $p=0.0472$, Figure S3). Overall, the models indicated that while the six universally mutated core genes were broadly responsible for vancomycin resistance in NRS70 and NRS384, they were of differential importance within each background. In addition, we did not find strong evidence for pairwise interaction between SNPs influencing MIC.

\section{Discussion}

In this project we looked for evidence of strain specificity in patterns of genetic change responsible for transition to vancomycin intermediate resistance in $S$. aureus. We found evidence 
396

397

398

399

400

401

402

403

404

405

406

407

408

409

410

411

412

413

414

415

416

417

418

419

420

421

422

423

424

425

426

427

428

429

430

431

432

433

434

435

that mutations in the same gene had differential effects on strain fitness and MIC depending on the genetic background. There were also statistically significant correlations in the patterns of cooccurrence of mutations linked to VISA. We did not probe the mechanisms of the putative epistatic interactions in this study. It is possible that, since many VISA-linked genes affect global cellular regulatory pathways, there is cross-talk with fixed mutations in the strain background that set global state changes (Priest et al., 2012). The strains also differ in their accessory gene content (Lindsay et al., 2006; McCarthy \& Lindsay, 2010; Aanensen et al., 2016), which may also affect gene expression and protein interactions responsible for the VISA phenotype. This work adds to a growing body of literature on the importance of strain background in this pathogen. S. aureus lineages have been shown to be heterogeneous in several significant clinically relevant phenotypes such as toxin production, biofilm formation, and host immune resistance (King et al., 2016; Su et al., 2020). Epistatic interactions between antibiotic resistance and toxicity have also been explored (Yokoyama et al., 2018).

VISA was found to be linked to mutations (primarily SNPs) in a limited number of genes (e.g., walKR, rpoB/C, vraTSR). Six previously implicated VISA-associated genes (walK, prs, rpoB, $r p o C, v r a S, y v q F)$ (Howden et al., 2010) were mutated in our three genetic backgrounds. walK followed by $y v q F$ were the most commonly seen mutations. For $r p o B$, rpoC, vraS, and $y v q F$, NRS70 and NRS384 mutation incidence were most different, and NRS123 mutation incidence was intermediate between the others. These results may be partially explained by the closer genetic relationship between $\mathrm{CC} 1$ and CC8 than CC5 and CC8 (Petit \& Read, 2018). Other genes were found to have been mutated in two of the three genetic backgrounds and may have a role in vancomycin resistance or act as compensatory mutations. Several are already validated as being associated with vancomycin resistance (walR (Howden et al., 2011), vraR (Kato et al., 2010; Baek et al., 2017; Asadpour \& Ghazanfari, 2019), $\operatorname{mprF}$ (Ruzin et al., 2003; Chen et al., 2018)). NRS70 and NRS384 backgrounds had more of these genes in common than with NRS123, indicating that NRS70 and NRS384 strains may experience more similar selective pressures in the larger mutational landscape outside of the previously discussed six genes.

The overall vancomycin resistance levels achieved after experimental evolution in NRS70 and NRS384 VISA strains were similar, suggesting both backgrounds are equally capable of achieving lower and higher levels of vancomycin intermediate resistance. Linear regression demonstrated that genetic predictors of vancomycin resistance differed between backgrounds. walK, rpoC, vraS, and $y v q F$ were all significantly associated with vancomycin MICs in NRS70, with $v r a S$ and $y v q F$ contributing most significantly, but only walK was significant in NRS384. As NRS384 strains carried more mutations than the other two backgrounds, this may indicate that while a subset of the six universally mutated genes (walK, prs, rpoB, rpoC, vraS, yvqF) are necessary for vancomycin resistance, other genes may play a significant role in determining the level of vancomycin resistance achieved in NRS384 VISA strains but not NRS70 strains. 
436 We found that vancomycin-intermediate resistance imposed a fitness cost (measured by maximal 437 growth rate compared to parent strain) to $S$. aureus that linearly scaled with the level of

438

439

440

441

442

443

444

445

446

447

448

449

450

451

452

453

454

455

456

457

458

459

460

461

462

463

464

465

466

467

468

469

470

471

472

473

474

475 vancomycin resistance in most cases. Fitness distributions between these two genetic backgrounds were markedly different at stages $4 \mu \mathrm{g} / \mathrm{mL}$ and $6 \mu \mathrm{g} / \mathrm{mL}$, with vancomycin resistance imposing less of a fitness cost on NRS70 VISA strains than NRS384 VISA strains. This may have clinical implications as it suggests that NRS70 S. aureus strains may evolve vancomycin resistance quickly, not be rapidly selected against due to growth defects, and may reach fixation if vancomycin concentrations are sustained and lead to the extinction of the ancestral vancomycin susceptible strain.

The laboratory evolution approach used in this study gave us the power to evolve VISA multiple times independently and achieve statistical significance using general linear models. With these data, we were able to investigate the effect of different SNPs with the strain background as a fixed effect. However, we cannot draw strong conclusions about clinical relevance as clinical VISA strains are the result of within-host evolution, and immune pressure is not present within our experimental design. Other factors that are present in human infection that were omitted in our experiment include but are not limited to nutrient limiting conditions and microenvironments. These undoubtedly reduce the mutational space $S$. aureus has available for adaption. In addition, the mutations in our studies were grouped by gene for simplicity, but likely not all mutations in VISA genes contribute equally to vancomycin resistance, and some may have no effect. The sheer number of mutations makes it impossible to recapitulate these mutations individually. We did not run control evolution experiments without vancomycin, so we cannot say whether some of the mutations in the most common six gene were due to random genetic drift, although it is well established that VISA strains do not evolve from VSSA in the laboratory in the absence of antibiotic selection. Another study design limitation was that fitness determinations by measuring maximal growth rate do not always correlate with competitive indices of evolved strains to ancestor strains and have been shown to be sensitive to environmental conditions. Thus, our measured fitness values may not accurately reflect withinhost fitness, especially in environments with competing microbes.

The results of this study offer some encouragement that development of VISA strains may be predicted with reasonable accuracy directly from the genome sequence, an important future technique in diagnostic clinical microbiology ( $\mathrm{Su}$, Satola \& Read, 2019). This is because the most common VISA mutations occurred in all three backgrounds, suggesting a "dictionary" of common mutations could be compiled. However, the finding that individual gene contribution to MIC may be background dependent (Table 4) suggests that prediction of the level of resistance in strains from different clonal complexes may be a much harder problem. In order to obtain the data to make exhaustive catalogs of VISA mutations and their epistatic dependencies or to build an accurate classifier, much more extensive versions of the experiment approach outlined here need to be performed, with a greater number of representative genetic backgrounds. 
476

477

478

479

480

481

482

483

484

485

486

487

488

489

490

491

492

493

494

495

496

497

498

499

500

501

502

503

504

505

506

507

508

509

510

511

512

513

514

515

516

\section{Conclusions}

We found that there was a complex relationship between genetic background and VISA. There was clear evidence for parallel evolution of VISA through mutations in a common set of genes across strains (especially: walK, prs, rpoB, rpoC, vraS, yvqF). Some of these genes (particularly $y v q F$ and $v r a S$ showed negative epistasis in all strains. However, we found evidence the effects of mutants on vancomycin MIC and the relationship between fitness and MIC were dependent on strain background. These results indicate that prediction of the levels of vancomycin resistance based on genome sequence may require extensive databases of mutants from different genetic backgrounds.

\section{Acknowledgements}

We would like to thank Jon Moller and Robert Petit for discussion and comments on the manuscript as well as Sujith Swarna for help with the experimental evolution experiments.

\section{References}

Aanensen DM, Feil EJ, Holden MTG, Dordel J, Yeats CA, Fedosejev A, Goater R, Castillo-Ramírez S, Corander J, Colijn C, Chlebowicz MA, Schouls L, Heck M, Pluister G, Ruimy R, Kahlmeter G, Åhman J, Matuschek E, Friedrich AW, Parkhill J, Bentley SD, Spratt BG, Grundmann H, European SRL Working Group. 2016. Whole-Genome Sequencing for Routine Pathogen Surveillance in Public Health: a Population Snapshot of Invasive Staphylococcus aureus in Europe. mBio 7. DOI: 10.1128/mBio.00444-16.

Alam MT, Petit RA 3rd, Crispell EK 3rd, Thornton TA, Conneely KN, Jiang Y, Satola SW, Read TD. 2014. Dissecting vancomycin intermediate resistance in Staphylococcus aureus using genome-wide association. Genome biology and evolution. DOI: 10.1093/gbe/evu092.

Asadpour L, Ghazanfari N. 2019. Detection of vancomycin nonsusceptible strains in clinical isolates of Staphylococcus aureus in northern Iran. International microbiology: the official journal of the Spanish Society for Microbiology 22:411-417. DOI: 10.1007/s10123-019-00063-7.

Askarian F, Ajayi C, Hanssen A-M, van Sorge NM, Pettersen I, Diep DB, Sollid JUE, Johannessen M. 2016. The interaction between Staphylococcus aureus $\mathrm{SdrD}$ and desmoglein 1 is important for adhesion to host cells. Scientific reports 6:22134. DOI: 10.1038/srep22134.

Askarian F, Uchiyama S, Valderrama JA, Ajayi C, Sollid JUE, van Sorge NM, Nizet V, van Strijp JAG, Johannessen M. 2017. Serine-Aspartate Repeat Protein D Increases Staphylococcus aureus Virulence and Survival in Blood. Infection and immunity 85. DOI: 10.1128/IAI.00559-16.

Baba T, Takeuchi F, Kuroda M, Yuzawa H, Aoki K-I, Oguchi A, Nagai Y, Iwama N, Asano K, Naimi T, Kuroda H, Cui L, Yamamoto K, Hiramatsu K. 2002. Genome and virulence determinants of high virulence community-acquired MRSA. The Lancet 359:1819-1827.

Baek JY, Chung DR, Ko KS, Kim SH, Yang S-J, Kang C-I, Peck KR, Song J-H. 2017. Genetic alterations responsible for reduced susceptibility to vancomycin in community-associated MRSA strains of ST72. The Journal of antimicrobial chemotherapy 72:2454-2460. DOI: 
517 Barber KE, Ireland CE, Bukavyn N, Rybak MJ. 2014. Observation of "seesaw effect" with vancomycin,

518

519

520

521

522

523

524

525

526

527

528

529

530

531

532

533

534

535

536

537

538

539

540

541

542

543

544

545

546

547

548

549

550

551

552

553

554

555

556

557

558

559

560 teicoplanin, daptomycin and ceftaroline in 150 unique MRSA strains. Infectious diseases and therapy 3:35-43. DOI: 10.1007/s40121-014-0023-0.

Barbu EM, Ganesh VK, Gurusiddappa S, Mackenzie RC, Foster TJ, Sudhof TC, Höök M. 2010. betaNeurexin is a ligand for the Staphylococcus aureus MSCRAMM SdrC. PLoS pathogens 6:e1000726. DOI: 10.1371/journal.ppat.1000726.

Barbu EM, Mackenzie C, Foster TJ, Höök M. 2014. SdrC induces staphylococcal biofilm formation through a homophilic interaction. Molecular microbiology 94:172-185. DOI: 10.1111/mmi.12750.

Belikova D, Jochim A, Power J, Holden MTG, Heilbronner S. 2020. "Gene accordions" cause genotypic and phenotypic heterogeneity in clonal populations of Staphylococcus aureus. Nature communications 11:3526. DOI: 10.1038/s41467-020-17277-3.

Boyle-Vavra S, Jones M, Gourley BL, Holmes M, Ruf R, Balsam AR, Boulware DR, Kline S, Jawahir S, Devries A, Peterson SN, Daum RS. 2011. Comparative genome sequencing of an isogenic pair of USA800 clinical methicillin-resistant Staphylococcus aureus isolates obtained before and after daptomycin treatment failure. Antimicrobial agents and chemotherapy 55:2018-2025. DOI: 10.1128/AAC.01593-10.

Bushnell B. 2016. BBMap Short Read Aligner. Berkeley: University of California, California. Available at http://sourceforge.net/projects/bbmap.

Camacho C, Coulouris G, Avagyan V, Ma N, Papadopoulos J, Bealer K, Madden TL. 2009. BLAST : architecture and applications. BMC Bioinformatics 10:421. DOI: 10.1186/1471-2105-10-421.

Cameron DR, Ward D V, Kostoulias X, Howden BP, Moellering RC, Eliopoulos GM, Peleg AY. 2012. Serine/threonine phosphatase Stp1 contributes to reduced susceptibility to vancomycin and virulence in Staphylococcus aureus. The Journal of infectious diseases 205:1677-1687. DOI:

10.1093/infdis/jis252.

Charles PGP, Ward PB, Johnson PDR, Howden BP, Grayson ML. 2004. Clinical features associated with bacteremia due to heterogeneous vancomycin-intermediate Staphylococcus aureus. Clinical infectious diseases: an official publication of the Infectious Diseases Society of America 38:448-451. DOI: $10.1086 / 381093$.

Cheng AG, Kim HK, Burts ML, Krausz T, Schneewind O, Missiakas DM. 2009. Genetic requirements for Staphylococcus aureus abscess formation and persistence in host tissues. FASEB journal: official publication of the Federation of American Societies for Experimental Biology 23:3393-3404. DOI: 10.1096/fj.09-135467.

Chen F-J, Lauderdale T-L, Lee C-H, Hsu Y-C, Huang I-W, Hsu P-C, Yang C-S. 2018. Effect of a Point Mutation in mprF on Susceptibility to Daptomycin, Vancomycin, and Oxacillin in an MRSA Clinical Strain. Frontiers in microbiology 9:1086. DOI: 10.3389/fmicb.2018.01086.

Clsi. 2013. Performance standards for antimicrobial susceptibility testing: CLSI approved standard M100S23.

Corrigan RM, Miajlovic H, Foster TJ. 2009. Surface proteins that promote adherence of Staphylococcus aureus to human desquamated nasal epithelial cells. BMC microbiology 9:22. DOI: 10.1186/14712180-9-22.

Cui L, Isii T, Fukuda M, Ochiai T, Neoh H-M, Camargo ILB da C, Watanabe Y, Shoji M, Hishinuma T, Hiramatsu K. 2010. An RpoB mutation confers dual heteroresistance to daptomycin and vancomycin in Staphylococcus aureus. Antimicrobial agents and chemotherapy 54:5222-5233. DOI: 10.1128/AAC.00437-10. 
561

562

563

564

565

566

567

568

569

570

571

572

573

574

575

576

577

578

579

580

581

582

583

584

585

586

587

588

589

590

591

592

593

594

595

596

597

598

599

600

601

602

603

604

Cui L, Tominaga E, Neoh H-M, Hiramatsu K. 2006. Correlation between Reduced Daptomycin Susceptibility and Vancomycin Resistance in Vancomycin-Intermediate Staphylococcus aureus. Antimicrobial agents and chemotherapy 50:1079-1082. DOI: 10.1128/AAC.50.3.1079-1082.2006.

Deatherage DE, Barrick JE. 2014. Identification of mutations in laboratory-evolved microbes from nextgeneration sequencing data using breseq. Methods in molecular biology 1151:165-188. DOI: 10.1007/978-1-4939-0554-6_12.

Deresinski S. 2009. Vancomycin heteroresistance and methicillin-resistant Staphylococcus aureus. The Journal of infectious diseases 199:605-609. DOI: 10.1086/596630.

Diep BA, Gill SR, Chang RF, Phan TH, Chen JH, Davidson MG, Lin F, Lin J, Carleton HA, Mongodin EF, Sensabaugh GF, Perdreau-Remington F. 2006. Complete genome sequence of USA300, an epidemic clone of community-acquired meticillin-resistant Staphylococcus aureus. The Lancet 367:731-739. DOI: 10.1016/S0140-6736(06)68231-7.

El-Halfawy OM, Valvano MA. 2015. Antimicrobial heteroresistance: an emerging field in need of clarity. Clinical microbiology reviews 28:191-207. DOI: 10.1128/CMR.00058-14.

Eyre DW, De Silva D, Cole K, Peters J, Cole MJ, Grad YH, Demczuk W, Martin I, Mulvey MR, Crook DW, Walker AS, Peto TEA, Paul J. 2017. WGS to predict antibiotic MICs for Neisseria gonorrhoeae. The Journal of antimicrobial chemotherapy 72:1937-1947. DOI: 10.1093/jac/dkx067.

Foucault M-L, Courvalin P, Grillot-Courvalin C. 2009. Fitness cost of VanA-type vancomycin resistance in methicillin-resistant Staphylococcus aureus. Antimicrobial agents and chemotherapy 53:23542359. DOI: 10.1128/AAC.01702-08.

Gardete S, Tomasz A. 2014. Mechanisms of vancomycin resistance in Staphylococcus aureus. The Journal of clinical investigation 124:2836-2840. DOI: 10.1172/JCI68834.

Hafer C, Lin Y, Kornblum J, Lowy FD, Uhlemann A-C. 2012. Vancomycin-intermediate Staphylococcus aureus: the contribution of selected gene mutations in clinical isolates. Antimicrobial agents and chemotherapy:27. DOI: 10.1128/AAC.01139-12.

Hiramatsu K, Hanaki H, Ino T, Yabuta K, Oguri T, Tenover FC. 1997. Methicillin-resistant Staphylococcus aureus clinical strain with reduced vancomycin susceptibility. The Journal of antimicrobial chemotherapy 40:135-136.

Holland TL, Fowler VG Jr. 2011. Vancomycin minimum inhibitory concentration and outcome in patients with Staphylococcus aureus bacteremia: pearl or pellet? The Journal of infectious diseases 204:329-331. DOI: 10.1093/infdis/jir275.

Horne KC, Howden BP, Grabsch EA, Graham M, Ward PB, Xie S, Mayall BC, Johnson PDR, Grayson ML. 2009. Prospective comparison of the clinical impacts of heterogeneous vancomycinintermediate methicillin-resistant Staphylococcus aureus (MRSA) and vancomycin-susceptible MRSA. Antimicrobial agents and chemotherapy 53:3447-3452. DOI: 10.1128/AAC.01365-08.

Howden BP, Davies JK, Johnson PDR, Stinear TP, Grayson ML. 2010. Reduced Vancomycin Susceptibility in Staphylococcus aureus, Including Vancomycin-Intermediate and Heterogeneous Vancomycin-Intermediate Strains: Resistance Mechanisms, Laboratory Detection, and Clinical Implications. Clinical microbiology reviews 23:99-139. DOI: 10.1128/CMR.00042-09.

Howden BP, Johnson PDR, Ward PB, Stinear TP, Davies JK. 2006. Isolates with low-level vancomycin resistance associated with persistent methicillin-resistant Staphylococcus aureus bacteremia. Antimicrobial agents and chemotherapy 50:3039-3047. DOI: 10.1128/AAC.00422-06.

Howden BP, McEvoy CRE, Allen DL, Chua K, Gao W, Harrison PF, Bell J, Coombs G, Bennett-Wood V, Porter JL, Robins-Browne R, Davies JK, Seemann T, Stinear TP. 2011. Evolution of Multidrug

Peer] reviewing PDF | (2020:11:55692:2:1:NEW 17 Jun 2021) 
605

606

607

608

609

610

611

612

613

614

615

616

617

618

619

620

621

622

623

624

625

626

627

628

629

630

631

632

633

634

635

636

637

638

639

640

641

642

643

644

645

646

647

648

Resistance during Staphylococcus aureus Infection Involves Mutation of the Essential Two Component Regulator WalKR. PLoS pathogens 7:e1002359. DOI:

10.1371/journal.ppat.1002359.t006.

Howden BP, Peleg AY, Stinear TP. 2013. The evolution of vancomycin intermediate Staphylococcus aureus (VISA) and heterogenous-VISA. Infection, genetics and evolution: journal of molecular epidemiology and evolutionary genetics in infectious diseases:1-9. DOI: 10.1016/j.meegid.2013.03.047.

Howden BP, Stinear TP, Allen DL, Johnson PDR, Ward PB, Davies JK. 2008. Genomic analysis reveals a point mutation in the two-component sensor gene graS that leads to intermediate vancomycin resistance in clinical Staphylococcus aureus. Antimicrobial agents and chemotherapy 52:3755-3762. DOI: 10.1128/AAC.01613-07.

Hu J, Zhang X, Liu X, Chen C, Sun B. 2015. Mechanism of Reduced Vancomycin Susceptibility Conferred by walK Mutation in Community-Acquired Methicillin-Resistant Staphylococcus aureus Strain MW2. Antimicrobial agents and chemotherapy 59:1352-1355. DOI: 10.1128/AAC.04290-14. Johnson PJT, Levin BR. 2013. Pharmacodynamics, population dynamics, and the evolution of persistence in Staphylococcus aureus. PLoS genetics 9:e1003123. DOI: 10.1371/journal.pgen.1003123.

Josefsson E, McCrea KW, Ní Eidhin D, O’Connell D, Cox J, Höök M, Foster TJ. 1998. Three new members of the serine-aspartate repeat protein multigene family of Staphylococcus aureus. Microbiology 144 ( Pt 12):3387-3395. DOI: 10.1099/00221287-144-12-3387.

Katayama Y, Azechi T, Miyazaki M, Takata T, Sekine M, Matsui H, Hanaki H, Yahara K, Sasano H, Asakura K, Takaku T, Ochiai T, Komatsu N, Chambers HF. 2017. Prevalence of Slow-Growth Vancomycin Nonsusceptibility in Methicillin-Resistant Staphylococcus aureus. Antimicrobial agents and chemotherapy 61. DOI: 10.1128/AAC.00452-17.

Kato Y, Suzuki T, Ida T, Maebashi K. 2010. Genetic changes associated with glycopeptide resistance in Staphylococcus aureus: predominance of amino acid substitutions in YvqF/VraSR. The Journal of antimicrobial chemotherapy 65:37-45. DOI: 10.1093/jac/dkp394.

King JM, Kulhankova K, Stach CS, Vu BG, Salgado-Pabón W. 2016. Phenotypes and Virulence among Staphylococcus aureus USA100, USA200, USA300, USA400, and USA600 Clonal Lineages. mSphere 1. DOI: 10.1128/mSphere.00071-16.

Klevens RM, Morrison MA, Nadle J, Petit S, Gershman K, Ray S, Harrison LH, Lynfield R, Dumyati G, Townes JM, Craig AS, Zell ER, Fosheim GE, McDougal LK, Carey RB, Fridkin SK, Active Bacterial Core surveillance (ABCs) MRSA Investigators. 2007. Invasive methicillin-resistant Staphylococcus aureus infections in the United States. JAMA: the journal of the American Medical Association 298:1763-1771. DOI: 10.1001/jama.298.15.1763.

Kobayashi SD, Musser JM, DeLeo FR. 2012. Genomic analysis of the emergence of vancomycinresistant Staphylococcus aureus. mBio 3. DOI: 10.1128/mBio.00170-12.

Kuroda M, Ohta T, Uchiyama I, Baba T, Yuzawa H, Kobayashi I, Cui L, Oguchi A, Aoki K, Nagai Y, Lian J, Ito T, Kanamori M, Matsumaru H, Maruyama A, Murakami H, Hosoyama A, Mizutani-Ui Y, Takahashi NK, Sawano T, Inoue R, Kaito C, Sekimizu K, Hirakawa H, Kuhara S, Goto S, Yabuzaki J, Kanehisa M, Yamashita A, Oshima K, Furuya K, Yoshino C, Shiba T, Hattori M, Ogasawara N, Hayashi H, Hiramatsu K. 2001. Whole genome sequencing of meticillin-resistant Staphylococcus aureus. The Lancet 357:1225-1240.

Lalueza A, Chaves F, San Juan R, Daskalaki M, Otero JR, Aguado JM. 2010. Is high vancomycin minimum inhibitory concentration a good marker to predict the outcome of methicillin-resistant

PeerJ reviewing PDF | (2020:11:55692:2:1:NEW 17 Jun 2021) 
649

650

651

652

653

654

655

656

657

658

659

660

661

662

663

664

665

666

667

668

669

670

671

672

673

674

675

676

677

678

679

680

681

682

683

684

685

686

687

688

689

690

691

Staphylococcus aureus bacteremia? The Journal of infectious diseases 201:311-2; author reply 3123. DOI: $10.1086 / 649572$.

Lindsay JA, Moore CE, Day NP, Peacock SJ, Witney AA, Stabler RA, Husain SE, Butcher PD, Hinds J. 2006. Microarrays reveal that each of the ten dominant lineages of Staphylococcus aureus has a unique combination of surface-associated and regulatory genes. Journal of bacteriology 188:669676. DOI: 10.1128/JB.188.2.669-676.2006.

Liu C, Chambers HF. 2003. Staphylococcus aureus with heterogeneous resistance to vancomycin: epidemiology, clinical significance, and critical assessment of diagnostic methods. Antimicrobial agents and chemotherapy 47:3040-3045.

Liu H, Lv J, Qi X, Ding Y, Li D, Hu L, Wang L, Yu F. 2015. The carriage of the serine-aspartate repeat protein-encoding sdr genes among Staphylococcus aureus lineages. The Brazilian journal of infectious diseases: an official publication of the Brazilian Society of Infectious Diseases 19:498502. DOI: 10.1016/j.bjid.2015.07.003.

Liu L, Wang Y, Bojer MS, Andersen PS, Ingmer H. 2020. High persister cell formation by clinical Staphylococcus aureus strains belonging to clonal complex 30. Microbiology. DOI: 10.1099/mic.0.000926.

Machado H, Seif Y, Sakoulas G, Olson CA, Szubin R, Palsson BO, Nizet V, Feist AM. 2020. Environmental conditions dictate differential evolution of vancomycin resistance in Staphylococcus aureus. bioRxiv:2020.06.07.138933. DOI: 10.1101/2020.06.07.138933.

Maiques E, Ubeda C, Campoy S, Salvador N, Lasa I, Novick RP, Barbé J, Penadés JR. 2006. beta-lactam antibiotics induce the SOS response and horizontal transfer of virulence factors in Staphylococcus aureus. Journal of bacteriology 188:2726-2729. DOI: 10.1128/JB.188.7.2726-2729.2006.

Ma KC, Mortimer TD, Hicks AL, Wheeler NE, Sánchez-Busó L, Golparian D, Taiaroa G, Rubin DHF, Wang Y, Williamson DA, Unemo M, Harris SR, Grad YH. 2020. Increased antibiotic susceptibility in Neisseria gonorrhoeae through adaptation to the cervical environment. bioRxiv. DOI: 10.1038/s41467-020-17980-1.

Marlowe EM, Cohen MD, Hindler JF, Ward KW, Bruckner DA. 2001. Practical strategies for detecting and confirming vancomycin-intermediate Staphylococcus aureus: a tertiary-care hospital laboratory's experience. Journal of clinical microbiology 39:2637-2639. DOI:

10.1128/JCM.39.7.2637-2639.2001.

Matsuo M, Cui L, Kim J, Hiramatsu K. 2013. Comprehensive identification of mutations responsible for heterogeneous vancomycin-intermediate Staphylococcus aureus (hVISA)-to-VISA conversion in laboratory-generated VISA strains derived from hVISA clinical strain Mu3. Antimicrobial agents and chemotherapy 57:5843-5853. DOI: 10.1128/AAC.00425-13.

McCarthy AJ, Lindsay JA. 2010. Genetic variation in Staphylococcus aureus surface and immune evasion genes is lineage associated: implications for vaccine design and host-pathogen interactions. $B M C$ microbiology 10:173. DOI: 10.1186/1471-2180-10-173.

McEvoy CRE, Tsuji B, Gao W, Seemann T, Porter JL, Doig K, Ngo D, Howden BP, Stinear TP. 2013. Decreased vancomycin susceptibility in Staphylococcus aureus caused by IS256 tempering of WalKR expression. Antimicrobial agents and chemotherapy 57:3240-3249. DOI: 10.1128/AAC.00279-13.

McGuinness WA, Malachowa N, DeLeo FR. 2017. Vancomycin Resistance in Staphylococcus aureus. The Yale journal of biology and medicine 90:269-281.

Peer) reviewing PDF | (2020:11:55692:2:1:NEW 17 Jun 2021) 
692

693

694

695

696

697

698

699

700

701

702

703

704

705

706

707

708

709

710

711

712

713

714

715

716

717

718

719

720

721

722

723

724

725

726

727

728

729

730

731

732

733

734

Mwangi MM, Wu SW, Zhou Y, Sieradzki K, de Lencastre H, Richardson P, Bruce D, Rubin E, Myers E, Siggia ED, Tomasz A. 2007. Tracking the in vivo evolution of multidrug resistance in Staphylococcus aureus by whole-genome sequencing. Proceedings of the National Academy of Sciences of the United States of America 104:9451-9456. DOI: 10.1073/pnas.0609839104.

Nam EY, Yang S-J, Kim ES, Cho JE, Park K-H, Jung S-I, Yoon N, Kim D-M, Lee C-S, Jang H-C, Park Y, Lee KS, Kwak YG, Lee JH, Park SY, Hwang J-H, Kim M, Song K-H, Kim HB. 2018. Emergence of Daptomycin-Nonsusceptible Methicillin-Resistant Staphylococcus aureus Clinical Isolates Among Daptomycin-Naive Patients in Korea. Microbial drug resistance 24:534-541. DOI: 10.1089/mdr.2017.0212.

Neoh H-M, Cui L, Yuzawa H, Takeuchi F, Matsuo M, Hiramatsu K. 2008. Mutated response regulator graR is responsible for phenotypic conversion of Staphylococcus aureus from heterogeneous vancomycin-intermediate resistance to vancomycin-intermediate resistance. Antimicrobial agents and chemotherapy 52:45-53. DOI: 10.1128/AAC.00534-07.

Ohta T, Hirakawa H, Morikawa K, Maruyama A, Inose Y, Yamashita A, Oshima K, Kuroda M, Hattori M, Hiramatsu K, Kuhara S, Hayashi H. 2004. Nucleotide substitutions in Staphylococcus aureus strains, Mu50, Mu3, and N315. DNA research: an international journal for rapid publication of reports on genes and genomes 11:51-56.

Papkou A, Hedge J, Kapel N, Young B, Craig MacLean R. 2020. Efflux pump activity potentiates the evolution of antibiotic resistance across S. aureus isolates. Nature communications 11:1-15. DOI: 10.1038/s41467-020-17735-y.

Passalacqua KD, Satola SW, Crispell EK, Read TD. 2012. A Mutation in the PP2C Phosphatase Gene in a Staphylococcus aureus USA300 Clinical Isolate with Reduced Susceptibility to Vancomycin and Daptomycin. Antimicrobial agents and chemotherapy 56:5212-5223. DOI: 10.1128/AAC.05770-11.

Petit RA 3rd, Read TD. 2018. Staphylococcus aureus viewed from the perspective of 40,000+ genomes. PeerJ 6:e5261. DOI: 10.7717/peerj.5261.

Prakash, V, Lewis JS, Jorgensen JH. 2008. Vancomycin MICs for methicillin-resistant Staphylococcus aureus isolates differ based upon the susceptibility test method used. Antimicrobial agents and chemotherapy 52:4528. DOI: 10.1128/AAC.00904-08.

Priest NK, Rudkin JK, Feil EJ, van den Elsen JMH, Cheung A, Peacock SJ, Laabei M, Lucks DA, Recker M, Massey RC. 2012. From genotype to phenotype: can systems biology be used to predict Staphylococcus aureus virulence? Nature reviews. Microbiology 10:791-797. DOI: 10.1038/nrmicro2880.

R Core Team, R Foundation for Statistical Computing, Vienna, Austria. 2016.R: A language and environment for statistical computing

Ruzin A, Severin A, Moghazeh SL, Etienne J, Bradford PA, Projan SJ, Shlaes DM. 2003. Inactivation of $\mathrm{mprF}$ affects vancomycin susceptibility in Staphylococcus aureus. Biochimica et biophysica acta 1621:117-121. DOI: 10.1016/s0304-4165(03)00028-x.

Sabat A, Melles DC, Martirosian G, Grundmann H, van Belkum A, Hryniewicz W. 2006. Distribution of the serine-aspartate repeat protein-encoding sdr genes among nasal-carriage and invasive Staphylococcus aureus strains. Journal of clinical microbiology 44:1135-1138. DOI: 10.1128/JCM.44.3.1135-1138.2006.

Sakoulas G, Eliopoulos GM, Moellering RC Jr, Novick RP, Venkataraman L, Wennersten C, DeGirolami PC, Schwaber MJ, Gold HS. 2003. Staphylococcus aureus accessory gene regulator (agr) group II: is 
735

736

737

738

739

740

741

742

743

744

745

746

747

748

749

750

751

752

753

754

755

756

757

758

759

760

761

762

763

764

765

766

767

768

769

770

771

772

773

774

775

776

777

778

there a relationship to the development of intermediate-level glycopeptide resistance? The Journal of infectious diseases 187:929-938. DOI: 10.1086/368128.

Sakoulas G, Moellering RC. 2008. Increasing antibiotic resistance among methicillin-resistant Staphylococcus aureus strains. Clinical infectious diseases: an official publication of the Infectious Diseases Society of America 46 Suppl 5:S360-7. DOI: 10.1086/533592.

Satola SW, Caliendo AM, Farley MM, Patel JB, Burd EM. 2009. Lack of heteroresistance among Staphylococcus aureus isolates with vancomycin MICs of 2 micrograms per milliliter by automated testing. Journal of clinical microbiology 47:2680-2681. DOI: 10.1128/JCM.01184-09.

Schubert B, Maddamsetti R, Nyman J, Farhat MR, Marks DS. 2018. Genome-wide discovery of epistatic loci affecting antibiotic resistance in Neisseria gonorrhoeae using evolutionary couplings. Nature microbiology. DOI: 10.1038/s41564-018-0309-1.

Shoji M, Cui L, Iizuka R, Komoto A, Neoh H-M, Watanabe Y, Hishinuma T, Hiramatsu K. 2011. walK and clpP Mutations Confer Reduced Vancomycin Susceptibility in Staphylococcus aureus. Antimicrobial agents and chemotherapy 55:3870-3881. DOI: 10.1128/AAC.01563-10.

Sieradzki K, Tomasz A. 1999. Gradual alterations in cell wall structure and metabolism in vancomycinresistant mutants of Staphylococcus aureus. Journal of bacteriology 181:7566-7570. DOI: 10.1128/JB.181.24.7566-7570.1999.

Sitkiewicz I, Babiak I, Hryniewicz W. 2011. Characterization of transcription within sdr region of Staphylococcus aureus. Antonie van Leeuwenhoek 99:409-416. DOI: 10.1007/s10482-010-9476-7.

Sousa AL de, de Sousa AL, Maués D, Lobato A, Franco EF, Pinheiro K, Araújo F, Pantoja Y, da Silva AL da C, Morais J, Ramos RTJ. 2018. PhageWeb - Web Interface for Rapid Identification and Characterization of Prophages in Bacterial Genomes. Frontiers in Genetics 9. DOI: 10.3389/fgene.2018.00644.

Sprouffske K, Wagner A. 2016. Growthcurver: an R package for obtaining interpretable metrics from microbial growth curves. BMC bioinformatics 17:172. DOI: 10.1186/s12859-016-1016-7.

Su M, Lyles JT, Petit RA Iii, Peterson J, Hargita M, Tang H, Solis-Lemus C, Quave CL, Read TD. 2020. Genomic analysis of variability in Delta-toxin levels between Staphylococcus aureus strains. PeerJ 8:e8717. DOI: 10.7717/peerj.8717.

Su M, Satola SW, Read TD. 2019. Genome-Based Prediction of Bacterial Antibiotic Resistance. Journal of clinical microbiology 57. DOI: 10.1128/JCM.01405-18.

Swenson JM, Anderson KF, Lonsway DR, Thompson A, McAllister SK, Limbago BM, Carey RB, Tenover FC, Patel JB. 2009. Accuracy of Commercial and Reference Susceptibility Testing Methods for Detecting Vancomycin-Intermediate Staphylococcus aureus. Journal of clinical microbiology 47:2013-2017. DOI: 10.1128/JCM.00221-09.

Vaudaux P, Huggler E, Bernard L, Ferry T, Renzoni A, Lew DP. 2010. Underestimation of Vancomycin and Teicoplanin MICs by Broth Microdilution Leads to Under-detection of GlycopeptideIntermediate Isolates of Staphylococcus aureus. Antimicrobial agents and chemotherapy. DOI: 10.1128/AAC.00269-10.

Wang Y, Li X, Jiang L, Han W, Xie X, Jin Y, He X, Wu R. 2016. Novel Mutation Sites in the Development of Vancomycin- Intermediate Resistance in Staphylococcus aureus. Frontiers in microbiology 7:2163. DOI: 10.3389/fmicb.2016.02163.

Watanabe Y, Cui L, Katayama Y, Kozue K, Hiramatsu K. 2011. Impact of rpoB Mutations on Reduced Vancomycin Susceptibility in Staphylococcus aureus. Journal of clinical microbiology 49:26802684. DOI: 10.1128/JCM.02144-10.

Peer] reviewing PDF | (2020:11:55692:2:1:NEW 17 Jun 2021) 
779 Yokoyama M, Stevens E, Laabei M, Bacon L, Heesom K, Bayliss S, Ooi N, O’Neill AJ, Murray E, 780 Williams P, Lubben A, Reeksting S, Meric G, Pascoe B, Sheppard SK, Recker M, Hurst LD, Massey

781

782 RC. 2018. Epistasis analysis uncovers hidden antibiotic resistance-associated fitness costs hampering 784 the evolution of MRSA. Genome biology 19:94. DOI: 10.1186/s13059-018-1469-2.

Yoo JI, Kim JW, Kang GS, Kim HS, Yoo JS, Lee YS. 2013. Prevalence of amino acid changes in the yvqF, vraSR, graSR, and tcaRAB genes from vancomycin intermediate resistant Staphylococcus

785 aureus. The Journal of Microbiology 51:160-165. DOI: 10.1007/s12275-013-3088-7.

787

Zhang S, Sun X, Chang W, Dai Y, Ma X. 2015. Systematic Review and Meta-Analysis of the Epidemiology of Vancomycin-Intermediate and Heterogeneous Vancomycin-Intermediate

788 Staphylococcus aureus Isolates. PloS one 10:e0136082. DOI: 10.1371/journal.pone.0136082. 
Figure 1

Experimental evolution of a lineage VISA.

For each of the three $S$. aureus genetic backgrounds, up to 40 independent lineages were evolved from single colonies isolated from frozen culture streaks. The lineages were cultured in $\mathrm{BHI}$ broth containing increasing concentrations of vancomycin. At each stage, single colonies were isolated and frozen and tested for antibiotic MIC using Etest strips (vancomycin and daptomycin) and fitness using a growth curve based assays. Full details in the materials and methods section.

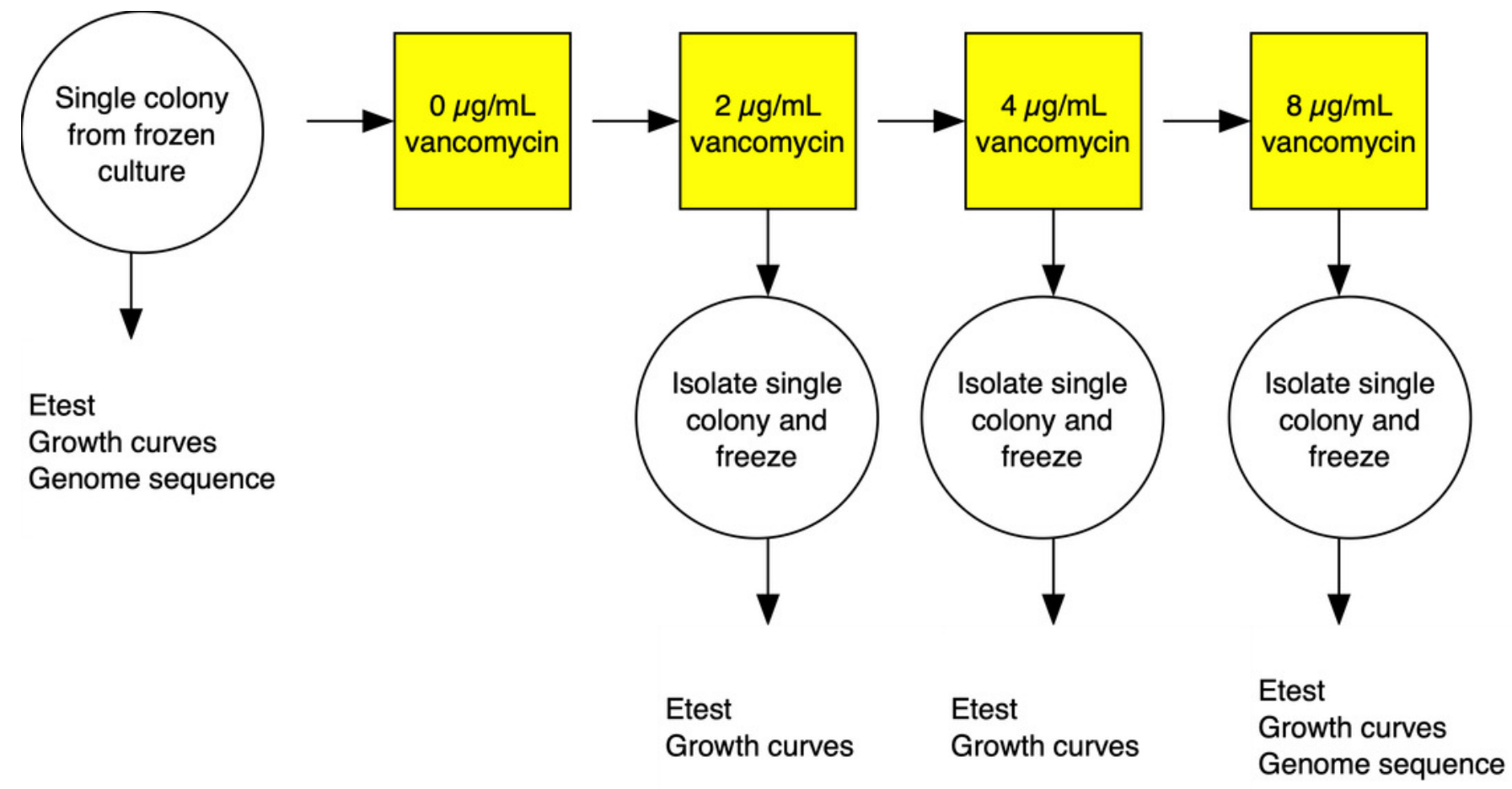




\section{Figure 2}

Correlation of mutations between VISA genes.

The numbers are correlation coefficients between genes based on the presence of nonsynonymous SNPs or frameshift deletions in the same strain (a) Correlation matrix of all evolved VISA strains. (b) Correlation matrix of all NRS70 evolved VISA strains. (c) Correlation matrix of all NRS123 evolved VISA strains. (d) Correlation matrix of all NRS384 evolved VISA strains. Significance codes: ' $* * * *^{\prime}<0.0001^{\prime} * *^{\prime}<0.001^{\prime} *^{\prime}<0.05$.

A

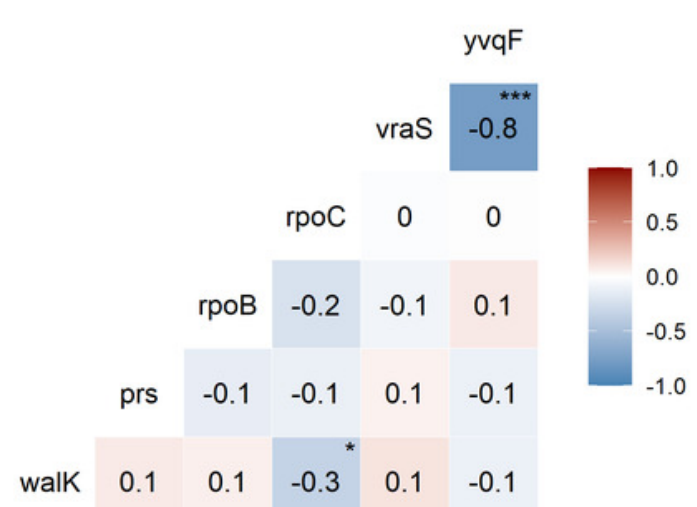

C

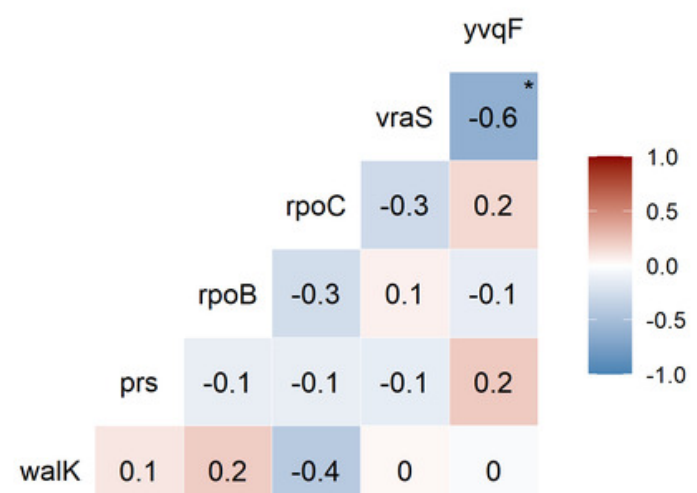

B

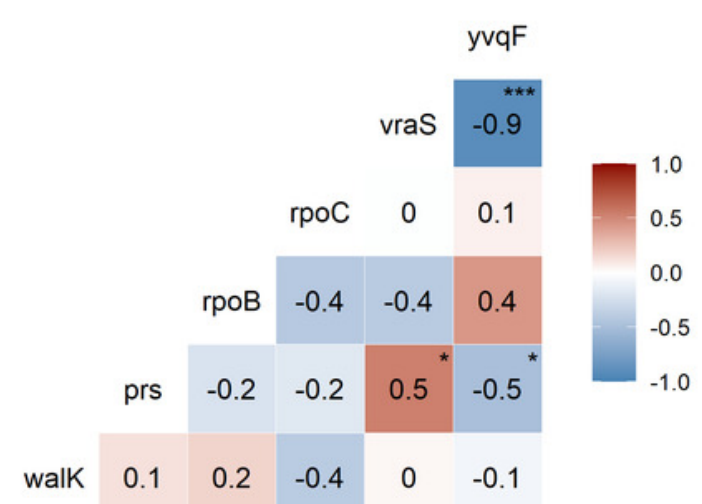

D

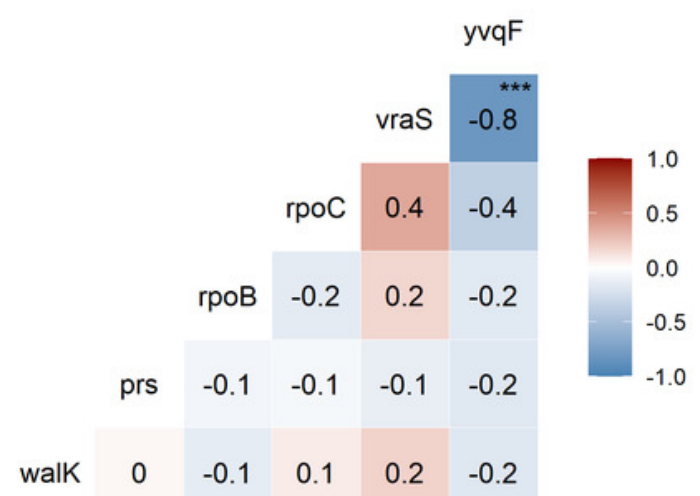


Figure 3

SNP distributions of VISA evolved strains differ by genetic background.

Mutations were called with breseq. Duplicate sdr mutations have been removed. Mode and median of SNPs per background: NRS70 (4), NRS123 (3), NRS384 (5). Significance codes: ${ }^{\prime} * * *^{\prime}<0.0001^{\prime} * *^{\prime}<0.001^{\prime} *^{\prime}<0.05$

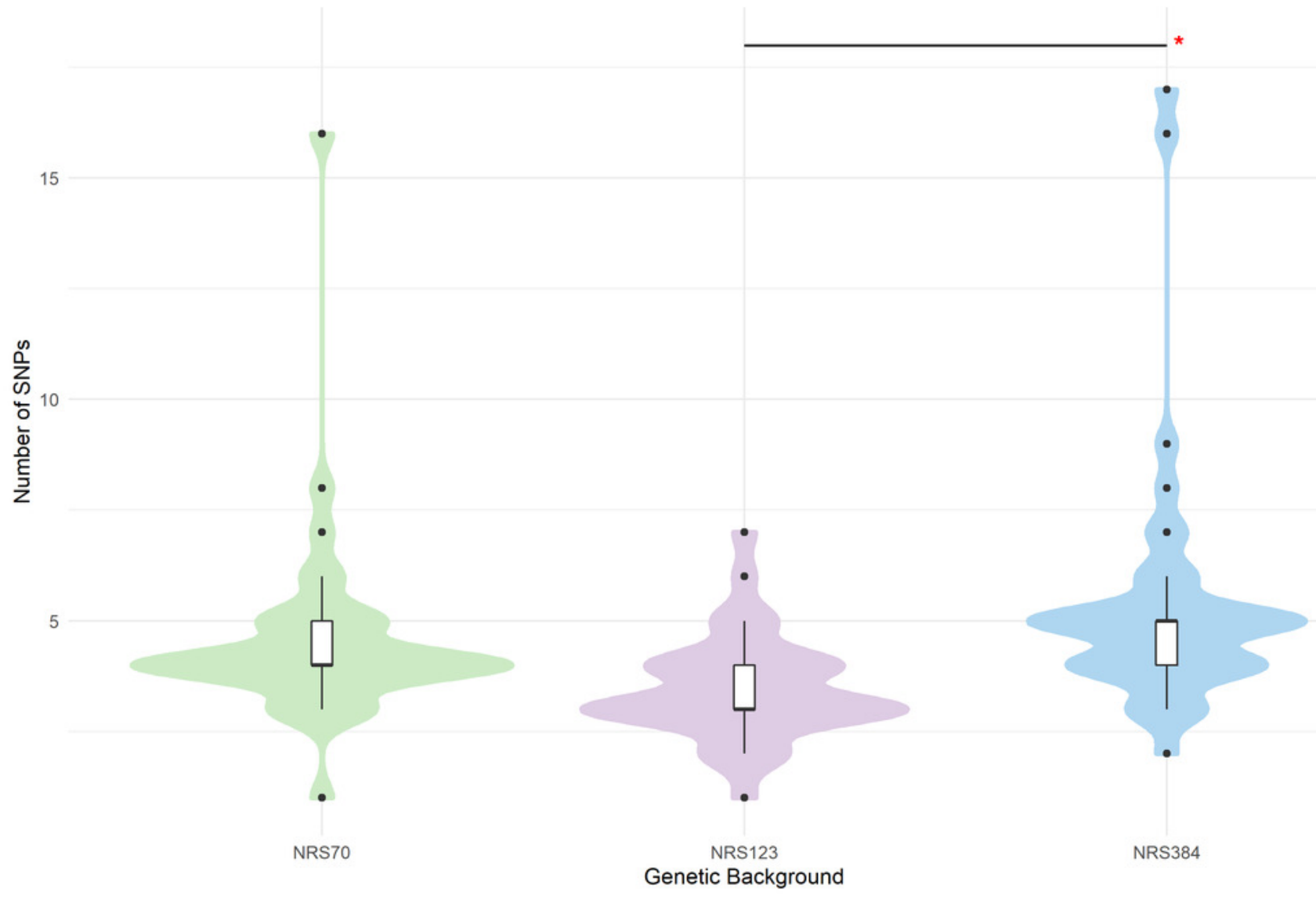




\section{Figure 4}

Antibiotic MIC distributions of NRS70 and NRS384 evolved strains.

Vancomycin MICs were determined by BMD and daptomycin MICs by Etest. (a) Vancomycin MIC distributions. The comparison of the two distributions by a Kolmogorov-Smirnov test was not statistically significant $(p=.3236)$. Parental MICs are indicated by a red triangle at 1 $\mu \mathrm{g} / \mathrm{ml}$. (b) Daptomycin MIC distributions. The comparison of the two distributions by a Kolmogorov-Smirnov test was not statistically significant $(p=.9996)$. Parental MICs are indicated by a red triangle at $.38 \mathrm{ug} / \mathrm{ml}$

A

10.0

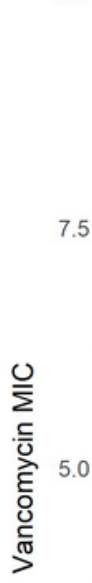

2.5

0.0

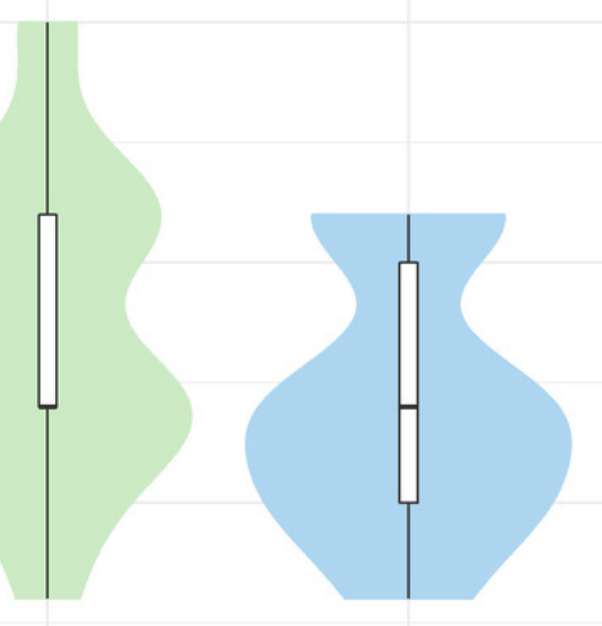

$\Delta$

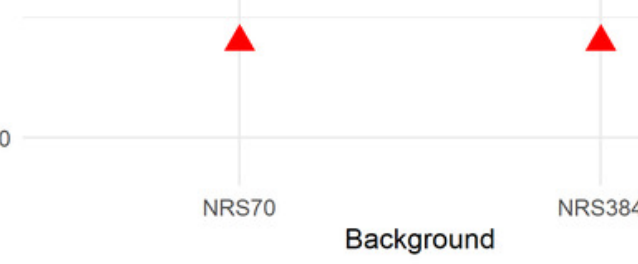

B

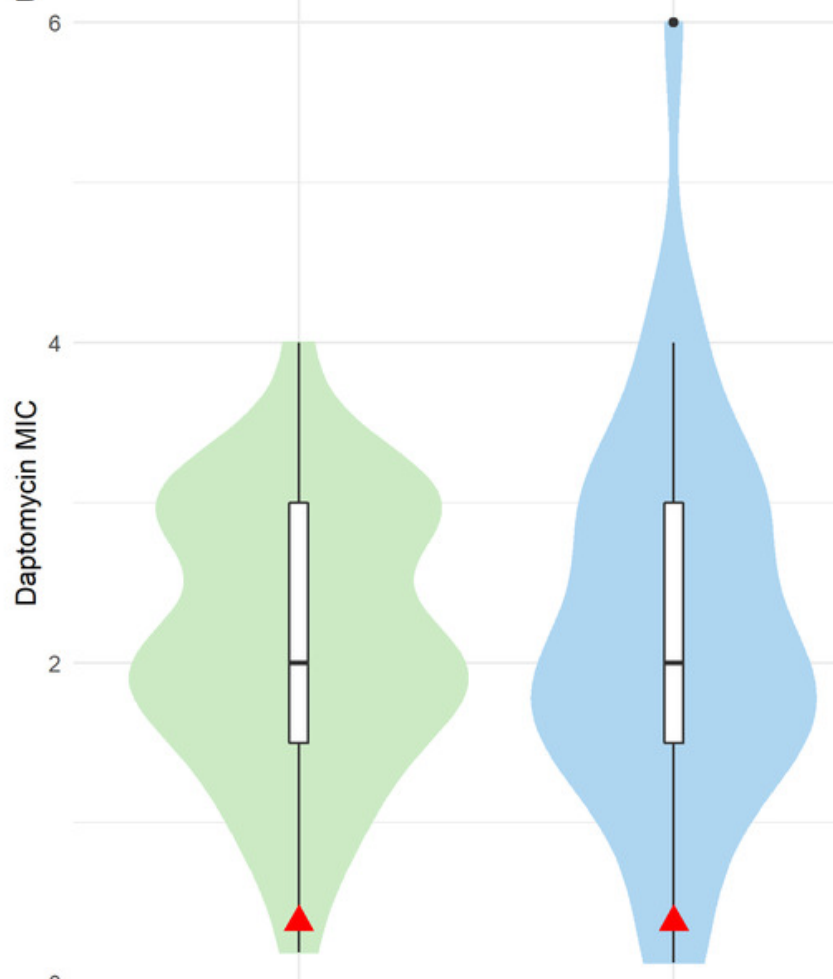

Background 


\section{Figure 5}

Linear relationship between vancomycin MIC and daptomycin MIC.

Vancomycin MICs were determined by BMD and daptomycin MICs by Etest. Linear regression of daptomycin MIC with vancomycin MIC was statistically significant $(p=.006)$. The comparison of the background specific linear relationships between vancomycin and daptomycin by an Analysis of Covariance (ANCOVA) was not statistically significant $(p=.822$ ). Overall: Adjusted R-squared: 0.08903 ( $p=.005)$; N70: Adjusted R-squared: 0.1866 ( $p=.005)$; N384: Adjusted R-squared: $0.03534(p=.13)$.

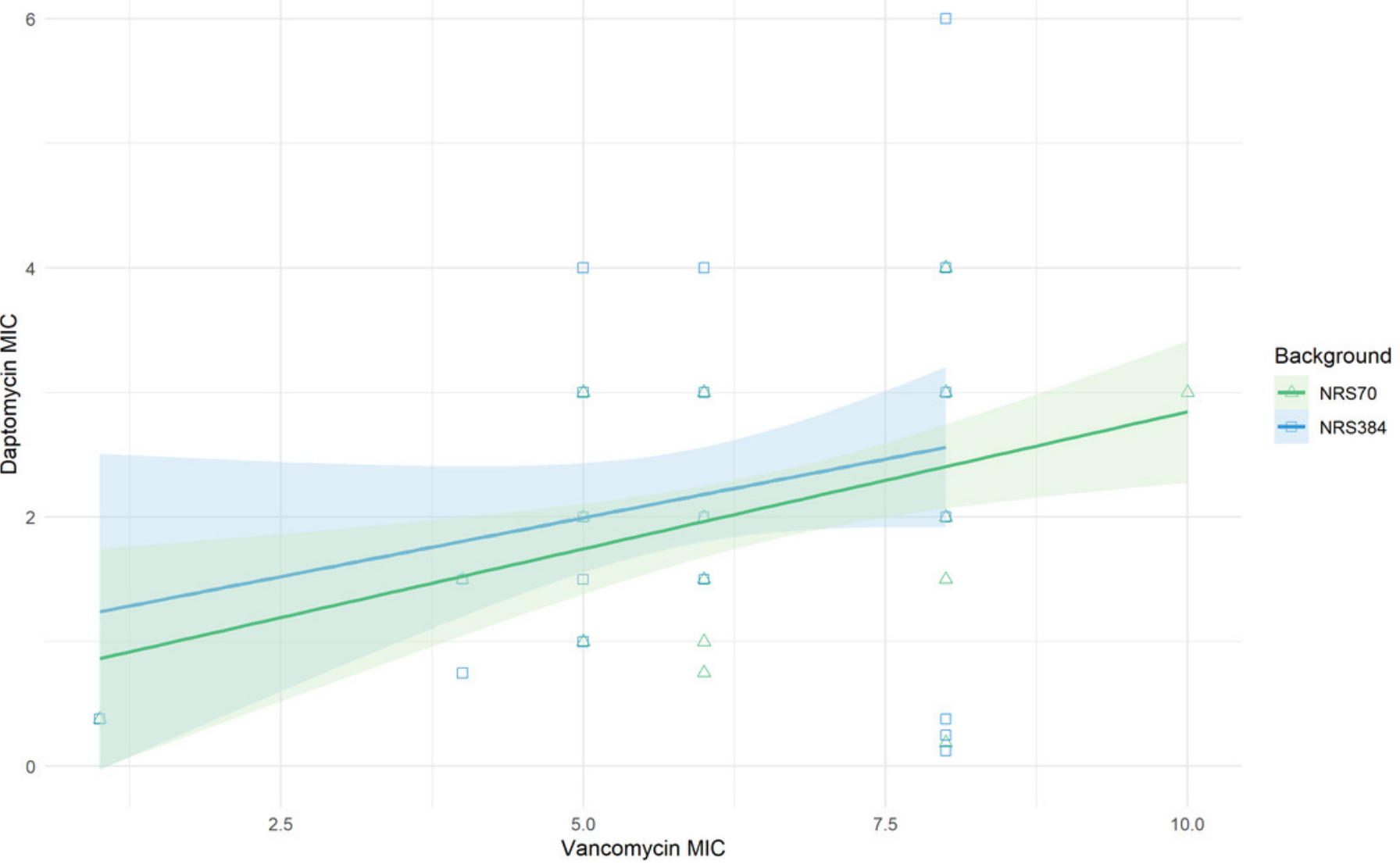




\section{Figure 6}

Fitness of NRS70 and NRS384 evolved strains.

Strains were grown in duplicate in a 96-well plate beginning at an OD $<.1$ and grown for 24 hours at $37^{\circ} \mathrm{C}$ with constant shaking in a Biotek Eon Microplate Spectrophotometer, with OD measurements every 10 minutes. To assess growth-curves, OD readings were imported into $\mathrm{R}$ and maximal growth rate $(\mathrm{r})$ calculated using the growthcurver package. Fitness was calculated as the ratio between the average $r$ of each evolved strain to the average $r$ of the parent strain. (a) Fitness ( $r$ ) of NRS70 evolved strains plotted against the concentration of vancomycin that the strains were evolved. NRS70-7 at vancomycin selection stage 6 and 8 and NRS70-14 at vancomycin selection stage 6 were excluded due to lack of growth or too large a variance between replicates. (b) Fitness ( $r$ ) progression of NRS384 evolved strains.
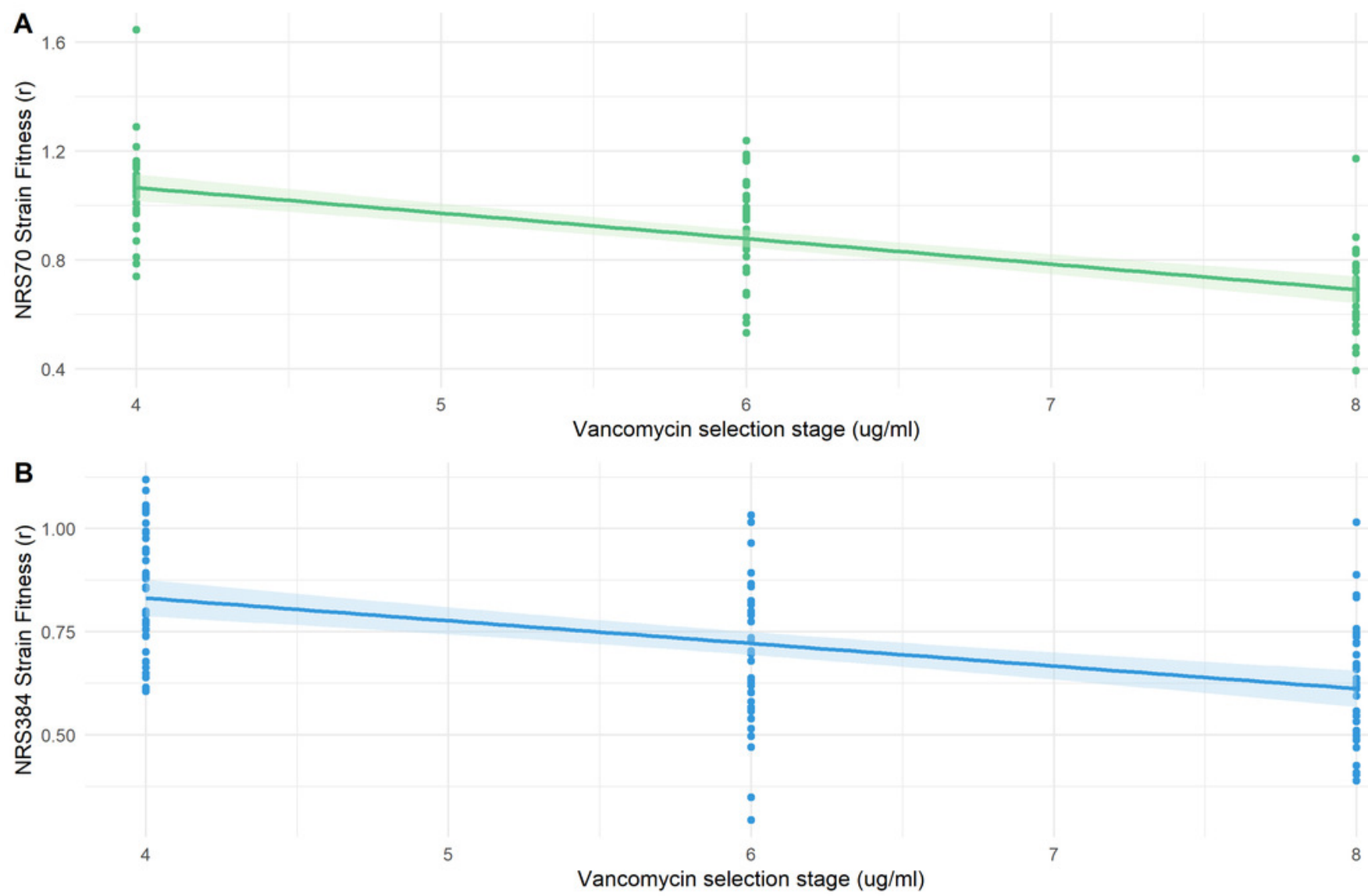


\section{Figure 7}

Fitness distributions at each stage of vancomycin selection for NRS70 and NRS384 strains.

MICs were determined by BMD. Strains were grown in duplicate in a 96-well plate beginning at an OD $<.1$ and grown for 24 hours at $37^{\circ} \mathrm{C}$ with constant shaking in a Biotek Eon Microplate Spectrophotometer, with OD measurements every 10 minutes. To assess growthcurves, $O D$ readings were imported into $R$ and maximal growth rate $(r)$ calculated using the growthcurver package. Fitness was calculated as the ratio between the average $r$ of each evolved strain to the average $r$ of the parent strain. Fitness distributions between NRS70 evolved VISA strains and NRS384 evolved VISA strains were compared using a two-sided twosample Kolmogorov-Smirnov test. (a) Vancomycin selection stage $4 \mu \mathrm{g} / \mathrm{mL}$. (b) Vancomycin selection stage $6 \mu \mathrm{g} / \mathrm{mL}$. (c) Vancomycin selection stage $8 \mu \mathrm{g} / \mathrm{mL}$. NRS70-7 at vancomycin selection stage 6 and 8 and NRS70-14 at vancomycin selection stage 6 were excluded due to lack of growth or too large a variance between replicates. 

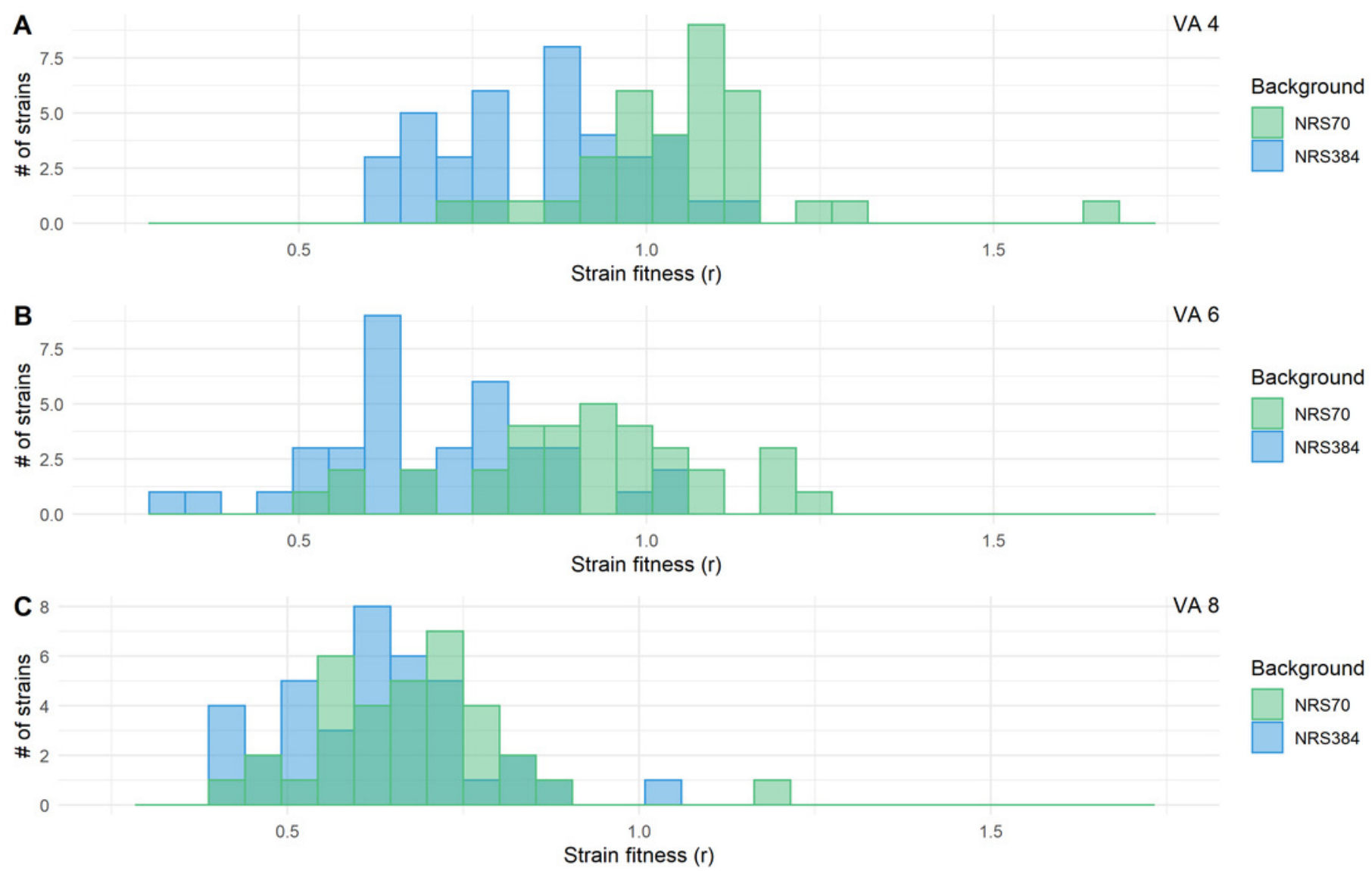

Background $\square$ NRS70 NRS384 


\section{Table 1 (on next page)}

Prevalence of mutations in six universally mutated genes by genetic background.

Mutations in these genes were called by breseq. The NRS70 walK prevalence calculation considers the presence of SNPs as well as inframe deletions. All other prevalence calculations are based solely on SNPs. 


\begin{tabular}{|l|l|l|l|}
\hline Gene / Background & NRS70 & NRS123 & NRS384 \\
\hline walK & $80 \%$ & $85.3 \%$ & $94.7 \%$ \\
\hline prs & $5.7 \%$ & $5.9 \%$ & $2.6 \%$ \\
\hline rpoB & $45.7 \%$ & $20.6 \%$ & $18.4 \%$ \\
\hline rpoC & $34.3 \%$ & $20.6 \%$ & $10.5 \%$ \\
\hline vraS & $17.1 \%$ & $23.6 \%$ & $39.5 \%$ \\
\hline yvqF & $80 \%$ & $55.9 \%$ & $55.3 \%$ \\
\hline
\end{tabular}

1 
Table 2 (on next page)

Genes with SNPs shared between two genetic backgrounds. 


\begin{tabular}{|c|c|c|c|}
\hline & NRS70 and NRS123 & NRS123 and NRS384 & NRS70 and NRS384 \\
\hline $\begin{array}{l}\text { Genes shared } \\
\text { in at least } 1 \\
\text { evolved } \\
\text { isolate }\end{array}$ & $\begin{array}{l}\text { WP_000101976.1 } \\
(w a l R) \\
\text { WP_000035320.1 } \\
(p d h A) \\
\text { WP_001032833.1 } \\
\text { (gad) } \\
\text { WP_000375864.1 } \\
\text { (HTH cro/C1-type } \\
\text { domain-containing } \\
\text { protein) } \\
\text { WP_000251253.1 } \\
\text { (UPF0374 protein) }\end{array}$ & $\begin{array}{l}\text { WP_001081640.1 } \\
\text { (Cyclic-di-AMP } \\
\text { phosphodiesterase) } \\
\text { WP_000260117.1 (pdhD) } \\
\text { WP_001071136.1 (mprF) } \\
\text { WP_000809131.1(cspA) } \\
\text { WP_000048060.1 (rpsU) } \\
\text { WP_000830380.1 (mgt) }\end{array}$ & $\begin{array}{l}\text { WP_000631969.1 } \\
(\text { cysS) } \\
\text { WP_001060462.1 } \\
(\text { sdrC) } \\
\text { WP_001273060.1 } \\
(\text { tagG) } \\
\text { WP_000491755.1 } \\
(y k a A) \\
\text { WP_000120368.1 } \\
(\text { pitA) } \\
\text { WP_000431312.1 } \\
(g r e A) \\
\text { WP_000782121.1 } \\
(p r s A) \\
\text { WP_000153535.1 } \\
(v r a R) \\
\text { WP_000159960.1 } \\
(p y r G) \\
\text { WP_000347896.1 } \\
(\text { dacA) } \\
\text { WP_000004085.1 } \\
(\text { rpsQ) } \\
\text { WP_000008673.1 } \\
(\text { ureC) }\end{array}$ \\
\hline
\end{tabular}




\section{Table 3 (on next page)}

Synonymous SNPs and nonsynonymous SNPs by genetic background.

Numbers given are the sum of the SNPs of all strains in that background (NRS70: 35 strains, NRS123: 34 strains, NRS384: 38 strains. The number of mutations when excluding sdr operon mutations is given in parenthesis. 


\begin{tabular}{|l|l|l|}
\hline & Synonymous & Nonsynonymous \\
\hline NRS70 & $19(10)$ & 149 \\
\hline NRS123 & 4 & 114 \\
\hline NRS384 & $45(32)$ & 166 \\
\hline
\end{tabular}

1 


\section{Table 4 (on next page)}

Linear regression models of vancomycin MIC.

An initial model was fitted using walK, prs, rpoB, rpoC, vraS, and yvqF as binary (mutated or not) predictors for log transformed vancomycin MICs. The predictors in the final were chosen by backwards selection. 


\begin{tabular}{|c|c|c|c|c|c|}
\hline Background & Gene & Estimate (initial) & p (initial) & Estimate (final) & p (final) \\
\hline NRS70 & $\begin{array}{l}\text { walK } \\
\text { prs } \\
\text { rpoB } \\
\text { rpoC } \\
\text { vraS } \\
\text { yvqF }\end{array}$ & $\begin{array}{l}.288 \\
.198 \\
.132 \\
.309 \\
.901 \\
.956\end{array}$ & $\begin{array}{l}.015 \\
.389 \\
.254 \\
.012 \\
.0005 \\
<10^{-4}\end{array}$ & $\begin{array}{l}.298 \\
- \\
- \\
.228 \\
.989 \\
1.058\end{array}$ & $\begin{array}{l}.012 \\
- \\
- \\
.026 \\
<10^{-4} \\
<10^{-5}\end{array}$ \\
\hline NRS384 & $\begin{array}{l}\text { walK } \\
\text { prs } \\
\text { rpoB } \\
\text { rpoC } \\
\text { vraS } \\
\text { yvqF }\end{array}$ & $\begin{array}{l}.629 \\
.255 \\
.103 \\
-.050 \\
.412 \\
.450\end{array}$ & $\begin{array}{l}.004 \\
.515 \\
.460 \\
.790 \\
.129 \\
.073\end{array}$ & $\begin{array}{l}.633 \\
- \\
- \\
- \\
.340 \\
.378\end{array}$ & $\begin{array}{l}.002 \\
- \\
- \\
- \\
.096 \\
.053\end{array}$ \\
\hline
\end{tabular}

1 